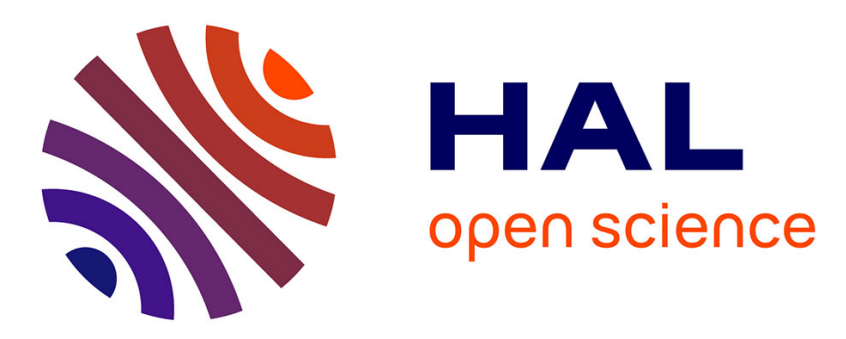

\title{
Finite volume scheme with local high order discretization of Hydrostatic equilibrium for Euler equations with external forces
}

\author{
Emmanuel Franck, Laura S. Mendoza
}

\section{- To cite this version:}

Emmanuel Franck, Laura S. Mendoza. Finite volume scheme with local high order discretization of Hydrostatic equilibrium for Euler equations with external forces. Journal of Scientific Computing, 2016, 10.1007/s10915-016-0199-4 . hal-01114437

\section{HAL Id: hal-01114437 \\ https://inria.hal.science/hal-01114437}

Submitted on 9 Feb 2015

HAL is a multi-disciplinary open access archive for the deposit and dissemination of scientific research documents, whether they are published or not. The documents may come from teaching and research institutions in France or abroad, or from public or private research centers.
L'archive ouverte pluridisciplinaire HAL, est destinée au dépôt et à la diffusion de documents scientifiques de niveau recherche, publiés ou non, émanant des établissements d'enseignement et de recherche français ou étrangers, des laboratoires publics ou privés. 


\title{
Finite volume scheme with local high order discretization of Hydrostatic equilibrium for Euler equations with external forces
}

\author{
E. Franck ${ }^{*}$ L. S. Mendoza ${ }^{\dagger \ddagger}$
}

February 9, 2015

\begin{abstract}
In this note, we introduce a new finite volume scheme for Euler equations with source terms: the gravity and the friction. The classical finite volume schemes are not able to capture correctly the dynamic induced by the balance between convective terms and external forces. Firstly, by plugging the source terms in the fluxes with the Jin-Levermore procedure, we modify the Lagrangian+remap scheme to obtain a method able to capture the asymptotic limit induced by the friction (asymptotic preserving scheme) and discretize with good accuracy the steady state linked to the gravity (well-balanced scheme). Secondly we will give some properties about this scheme and introduce a modification which allows us to obtain an arbitrary high order discretization of the hydrostatic steady state.
\end{abstract}

\section{Introduction}

In many applications, like gas dynamics [9], astrophysics [26], chemotaxis [24] or neutronic and photonic [6], the hyperbolic systems with source terms appear. Indeed, these systems modelize the balance between the convective effects, the acoustic effects and the external forces. A classical example of this type of problem is the Euler equations with friction and gravity used for the astrophysics applications (for example atmospheric phenomena) and is an interesting nonlinear model to begin the study of more complicated multi-fluid and multi-phases flows

$$
\left\{\begin{array}{l}
\partial_{t} \rho+\operatorname{div}(\rho \mathbf{u})=0 \\
\partial_{t} \rho u+\operatorname{div}(\rho \mathbf{u} \otimes \mathbf{u})+\nabla p=-\rho \nabla \phi-\sigma \rho \mathbf{u} \\
\partial_{t} \rho e+\operatorname{div}(\rho \mathbf{u} e)+\operatorname{div}(p \mathbf{u})=-\rho(\nabla \phi \cdot \mathbf{u})-\sigma \rho\|\mathbf{u}\|^{2}
\end{array}\right.
$$

with $\phi$ the gravity potential in general given by the elliptic equation $\triangle \phi=4 \pi \rho G$. The presence of the source terms can be modify the behavior of the model. In this work, we are interested subsets of solutions induced by the balanced between the hyperbolic system and the source terms. To analyze these subset of solutions we introduce the following scaling $\bar{t}=\varepsilon^{\alpha} t$ and $\bar{\sigma}=\frac{\sigma}{\varepsilon^{\beta}}$ (with $\gamma=\alpha+\beta)$. Plugging this scaling in the Euler equation

$$
\left\{\begin{array}{l}
\partial_{t} \rho+\frac{1}{\varepsilon^{\alpha}} \operatorname{div}(\rho \mathbf{u})=0 \\
\partial_{t} \rho u+\frac{1}{\varepsilon^{\alpha}} \operatorname{div}(\rho \mathbf{u} \otimes \mathbf{u})+\frac{1}{\varepsilon^{\alpha}} \nabla p=-\frac{1}{\varepsilon^{\alpha}} \rho \nabla \phi-\frac{\sigma}{\varepsilon^{\gamma}} \rho \mathbf{u} \\
\partial_{t} \rho e+\frac{1}{\varepsilon^{\alpha}} \operatorname{div}(\rho \mathbf{u} e)+\frac{1}{\varepsilon^{\alpha}} \operatorname{div}(p \mathbf{u})=-\frac{1}{\varepsilon^{\alpha}} \rho(\nabla \phi \cdot \mathbf{u})-\frac{\sigma}{\varepsilon^{\gamma}} \rho\|\mathbf{u}\|^{2}
\end{array}\right.
$$

\footnotetext{
*Inria Nancy grand Est, TONUS Team, Strasbourg, 67000, France

$\dagger^{\dagger}$ Max-Planck-Institut für Plasmaphysik, Boltzmannstraße 2, D-85748 Garching, Germany

‡Technische Universität München, Boltzmannstr. 3, D-85748 Garching, Germany.
} 
At the numerical level, it is known that the classical schemes are not efficient to capture the behavior induced by the balance between the source terms and the hyperbolic part. Since some years, specific numerical methods have been designed, in particular the asymptotic preserving schemes which capture the asymptotic limit independently of the relaxation parameter $\varepsilon$ and well-balanced schemes which discretize the steady states with a high accuracy.

The system (2) satisfies the following properties:

- The entropy inequality $\partial_{t}(\rho S)+\operatorname{div}(\rho \mathbf{u} S) \geq 0$, is satisfied for weak solutions

- For $\alpha=1$ and $\gamma=2$, when $\varepsilon$ tends to zero the system tends to

$$
\left\{\begin{array}{l}
\partial_{t} \rho+\operatorname{div}(\rho \mathbf{u})=0 \\
\partial_{t} \rho e+\operatorname{div}(\rho \mathbf{u} e)+p \operatorname{div}(\mathbf{u})=0 \\
\mathbf{u}=-\left(\frac{1}{\sigma}\left(\nabla \phi+\frac{\nabla p}{\rho}\right)\right) .
\end{array}\right.
$$

- For $\gamma=1, \alpha=0$ when $\varepsilon$ tends to zero

$$
\left\{\begin{array}{l}
\rho(t, \mathbf{x})=\rho(t=0, \mathbf{x}) \\
\mathbf{u}(t, \mathbf{x})=\mathbf{0} \\
e(t, \mathbf{x})=e(t=0, \mathbf{x})
\end{array}\right.
$$

- For $\gamma=0, \alpha=1$, when $\varepsilon$ tends to zero

$$
\left\{\begin{array}{l}
\mathbf{u}(t, \mathbf{x})=\mathbf{0} \\
\nabla p(t, \mathbf{x})=-\rho(t, \mathbf{x}) \nabla \phi(\mathbf{x})
\end{array}\right.
$$

The first subset of solutions correspond to the long time limit and high friction limit. In this case, the presence of the friction modify the dynamic of the solutions which can approximate the diffusion solutions of (3). The second subset of solutions correspond to the high friction limit which generate steady solution since the velocity is null for all time. The last subset of solutions corresponds to the long time limit with $\mathbf{u}=\mathbf{0}$. We are interested by these solutions called "hydrostatic equilibirum" which are important for astrophysics simulations, but we does not consider the long time solutions for $\mathbf{u}=$ cts. In this work, we propose to design a scheme which captures and preserves these solutions at the discrete level. In general, to discretize a hyperbolic nonlinear system, as the Euler equations, one uses first order Godunov-Type schemes (i.e. Rusanov scheme, entropic Roe scheme, Lagrange+remap schemes, etc.). These types of method satisfy by construction the entropic inequality and therefore the positivity of the density and the energy. Additionally, their stability and robustness to approximate the discontinuous solutions, have make them very popular. Nonetheless, for relaxation models with dissipative source terms which generate an asymptotic limit, these methods are not very adapted. Indeed, at the end one obtains a consistency error homogeneous to $O\left(\frac{\Delta x}{\varepsilon^{\alpha}}\right)$ (which comes from the numerical viscosity) and the CFL condition is constrained by $\varepsilon$. To solve the problem, some techniques have been introduced to modify the scheme in order to construct asymptotic preserving (AP) methods. The latter conserves the same property of stability (dissipation of entropy, positivity) but admits a consistency error homogeneous to $O(\Delta x)$ and its CFL condition is not constrained by $\varepsilon$. Another problem comes from the discretization of the steady state (hydrostatic equilibrium of high friction limit in this case). This is specially important in some applications, like astrophysical flows, where approximating with good accuracy the steady state is essential. For example, when we consider flows with very small velocity, the numerical error generates spurious velocities (homogeneous to $O(\Delta x)$ ) which are larger than the physical velocities. In this case, it is difficult to correctly capture the physical flows. To treat the discretization of these steady states, some technic called Well-Balanced schemes [20]-[14]-[15] have been introduced to discretize exactly of with a high accuracy these solutions. Some AP schemes [8]-9] and Well Balanced schemes [26] for Euler equations have been introduced recently. Observing the methods proposed in these papers we 
obtain that the "well balanced" and "asymptotic preserving" methods are chased on the same idea which consist to incorporate the source terms in the finite volumes schemes to capture the effects associated. Consequently using one method we propose to construct and analyze an adapted semi-implicit scheme (with a CFL independent of $\varepsilon$ ) for the Euler equation with friction and gravity which preserves the different subset of solutions defined by (3, 4 45 . Finally, we modify the method to obtain a good accuracy in the diffusion regime as well as in the steady states. In section 2, we introduce the scheme in $1 \mathrm{D}$, showing in details how to construct it as well as how the scheme conserves the AP properties. The final Well-Balanced scheme is presented, followed by some numerical results. An extension for $2 \mathrm{D}$ unstructured meshes can be found in section 3 where the modifications for the Euler equation and the high order discretization of the hydrostatic equilibrium 5 are presented. This $2 \mathrm{D}$ version was also numerically tested and the results can be found in the same section. At the end we use the same scheme to discretize the subset of solutions studied. However in the literature, the scheme which treat the diffusion solutions (3) are called "asymptotic preserving scheme" and the scheme which treat the diffusion solutions (4 50 are called "well-balanced scheme". Consequently in this paper we will talk of "asymptotic preserving property" for the diffusion solutions and "well-balanced property" for steady solutions. The scheme will be call "asymptotic preserving scheme".

\section{Scheme in 1D}

To construct the asymptotic preserving scheme we propose to use a one step Lagrange+remap (LR) scheme. The idea of the LR scheme is to solve firstly the equation in the Lagrangian formulation (fluid coordinates) on mobile mesh and after apply a projection on the first mesh to the quantities obtained at the end of the Lagrangian step. The Lagrangian fluxes (Lagrangian velocity and pressure at the interface) are computed using the linearized Riemann problem associated with the Lagrangian model. The remap scheme is equivalent to an advection of the conservative quantities at the Lagrangian velocity. Normally, for this scheme, the two steps are split. In our study we use a version without splitting where we sum the Lagrangian and the advection fluxes. We use this scheme because it is easy to separate the effect of the pressure terms (important for the asymptotic properties) and the convective terms.

\subsection{Construction of the scheme in 1D}

We consider an irregular 1D mesh. For the $j$-th cell, the nodes are denoted by $x_{j-\frac{1}{2}}$ and $x_{j+\frac{1}{2}}$, its middle point by $x_{j}$, its volume by $\Delta x_{j}=\left|x_{j+\frac{1}{2}}-x_{j-\frac{1}{2}}\right|$ and its dual volume by $\Delta x_{j+\frac{1}{2}}=\left|x_{j+1}-x_{j}\right|$. Thus, we can notice that $\frac{1}{2}\left(\Delta x_{j}+\Delta x_{j+1}\right)=\Delta x_{j+\frac{1}{2}}$. The quantities at a node correspond to the average value in the cell defined for a quantity $f$ by

$$
f_{j}=\frac{1}{\Delta x_{j}} \int_{x_{j-\frac{1}{2}}}^{x_{j+\frac{1}{2}}} f(t, x) d x
$$

For first and second order discretizations, we can associate the average value in the cell and the punctual value at the middle of the cell since $\left|f\left(x_{j}\right)-\frac{1}{\Delta x_{j}} \int_{x_{j-\frac{1}{2}}}^{x_{j+\frac{1}{2}}} f(t, x) d x\right| \leq C h^{2}$ with $h$ the step mesh.

Using these notations we can rewrite the system (2) as follows:

$$
\left\{\begin{array}{l}
\partial_{t} \rho_{j}+\frac{u_{j+\frac{1}{2}} \rho_{j+\frac{1}{2}}-u_{j-\frac{1}{2}} \rho_{j-\frac{1}{2}}}{\varepsilon^{\alpha} \Delta x_{j}}=0 \\
\partial_{t}(\rho u)_{j}+\frac{u_{j+\frac{1}{2}}(\rho u)_{j+\frac{1}{2}}-u_{j-\frac{1}{2}}(\rho u)_{j-\frac{1}{2}}}{\varepsilon^{\alpha} \Delta x_{j}}+\frac{p_{j+\frac{1}{2}}-p_{j-\frac{1}{2}}}{\varepsilon^{\alpha} \Delta x_{j}}=-\frac{1}{\varepsilon^{\alpha}}\left(\rho_{j}\left(\partial_{x} \phi\right)_{j}+\frac{\sigma}{\varepsilon^{\beta}} \rho_{j} u_{j}\right) \\
\partial_{t}(\rho e)_{j}+\frac{u_{j+\frac{1}{2}}(\rho e)_{j+\frac{1}{2}}-u_{j-\frac{1}{2}}(\rho e)_{j-\frac{1}{2}}}{\varepsilon^{\alpha} \Delta x_{j}}+\frac{p_{j+\frac{1}{2}} u_{j+\frac{1}{2}}-p_{j-\frac{1}{2}} u_{j-\frac{1}{2}}}{\varepsilon^{\alpha} \Delta x_{j}}=-\frac{1}{\varepsilon^{\alpha}}\left(\rho_{j} u_{j}\left(\partial_{x} \phi\right)_{j}+\frac{\sigma}{\varepsilon^{\beta}} \rho_{j} u_{j}^{2}\right)
\end{array}\right.
$$


with the Lagrangian fluxes defined by

$$
\left\{\begin{array}{l}
u_{j+\frac{1}{2}}=\frac{1}{2}\left(u_{j}+u_{j+1}\right)+\frac{1}{2 \rho c_{j+\frac{1}{2}}}\left(\rho_{j}-\rho_{j+1}\right) \\
p_{j+\frac{1}{2}}=\frac{1}{2}\left(\rho_{j}+\rho_{j+1}\right)+\frac{\rho c_{j+\frac{1}{2}}}{2}\left(u_{j}-u_{j+1}\right)
\end{array}\right.
$$

The upwind fluxes denoted by $f_{j+\frac{1}{2}}$ are defined by $f_{j+\frac{1}{2}}=f_{j}$ if $u_{j+\frac{1}{2}}>0$ and $f_{j+\frac{1}{2}}=f_{j+1}$ if $u_{j+\frac{1}{2}}<0$ with $f=\rho, \rho u$ or $\rho e$. The notation $\left(\partial_{x} \phi\right)_{j}$ corresponds to a discrete gradient of $\phi$ at the point $x_{j}$. The Lagrangian speed wave $\rho c_{j \pm \frac{1}{2}}$ is given by the arithmetic mean $\frac{1}{2}\left(\rho_{j} c_{j}+\rho_{j+1} c_{j+1}\right)$ or a non centred approximation $\rho c_{j \pm \frac{1}{2}}=\rho_{j} c_{j}$. Actually, this scheme is not asymptotic preserving nor well-balanced. To modify the scheme we propose to use the Jin-Levermore method [19] which consists to incorporate the source terms into the fluxes to capture the behaviour they induce. We begin by writing the Lagrangian fluxes on the following form

$$
\left\{\begin{array}{l}
p_{j+\frac{1}{2}}+\rho c_{j+\frac{1}{2}} u_{j+\frac{1}{2}}=p_{j}+\rho c_{j+\frac{1}{2}} u_{j} \\
p_{j+\frac{1}{2}}-\rho c_{j+\frac{1}{2}} u_{j+\frac{1}{2}}=p_{j+1}-\rho c_{j+\frac{1}{2}} u_{j+1}
\end{array}\right.
$$

This form corresponds to Riemann invariant associated to linearization of the Lagrangian system. Now, we want to introduce into the fluxes, the balance law between source terms and convective term which describe the asymptotic regime and the hydrostatic equilibrium.

The asymptotic analysis for the Euler equations shows that $u=O(\varepsilon)$. Thus we obtain an important relation for the asymptotic regime is $\rho u=\frac{\varepsilon^{\beta}}{\sigma}\left(-\rho g-\partial_{x} p\right)+o\left(\varepsilon^{\gamma}\right)$. To introduce this relation in the Lagrangian fluxes we propose to use the following equalities which come from a Taylor expansion:

$$
p\left(x_{j}\right) \approx p\left(x_{j+\frac{1}{2}}\right)-\frac{\Delta x_{j+\frac{1}{2}}}{2} \partial_{x} p\left(x_{j+\frac{1}{2}}\right) \text { and } p\left(x_{j+1}\right) \approx p\left(x_{j+\frac{1}{2}}\right)+\frac{\Delta x_{j+\frac{1}{2}}}{2} \partial_{x} p\left(x_{j+\frac{1}{2}}\right) .
$$

For the following we define a discrete quantity at the interface called $\left(\rho \partial_{x} \phi\right)_{j+\frac{1}{2}}$. We use these Taylor expansions the first order relation in $\varepsilon$ between the gradient of $p$ and the source terms and Lagrangian fluxes to obtain

$$
\left\{\begin{array}{l}
p_{j+\frac{1}{2}}+\rho c_{j+\frac{1}{2}} u_{j+\frac{1}{2}}-\frac{\Delta x_{j+\frac{1}{2}}}{2}\left(\left(\rho \partial_{x} \phi\right)_{j+\frac{1}{2}}+\frac{\sigma_{j+\frac{1}{2}}}{\varepsilon^{\beta}} \rho_{j+\frac{1}{2}} u_{j+\frac{1}{2}}\right)=p_{j}+\lambda_{j+\frac{1}{2}} u_{j} \\
p_{j+\frac{1}{2}}-\rho c_{j+\frac{1}{2}} u_{j+\frac{1}{2}}+\frac{\Delta x_{j+\frac{1}{2}}}{2}\left(\left(\rho \partial_{x} \phi\right)_{j+\frac{1}{2}}+\frac{\sigma_{j+\frac{1}{2}}}{\varepsilon^{\beta}} \rho_{j+\frac{1}{2}} u_{j+\frac{1}{2}}\right)=p_{j+1}-\rho c_{j+\frac{1}{2}} u_{j+1}
\end{array}\right.
$$

with $\rho_{j+\frac{1}{2}}=\frac{1}{2}\left(\rho_{j}+\rho_{j+1}\right)$ and $\left(\rho \partial_{x} \phi\right)_{j+\frac{1}{2}}$ approximations of $\rho \partial_{x} \phi$ at the interface. These two relations give the following fluxes

$$
\left\{\begin{array}{l}
p_{j+\frac{1}{2}}=\frac{1}{2}\left(p_{j}+p_{j+1}\right)+\frac{1}{2} \rho c_{j+\frac{1}{2}}\left(u_{j}-u_{j+1}\right) \\
u_{j+\frac{1}{2}}^{*}=M_{j+\frac{1}{2}}\left(\frac{1}{2}\left(u_{j}+u_{j+1}\right)+\frac{1}{2 \rho c_{j+\frac{1}{2}}}\left(p_{j}-p_{j+1}-\Delta x_{j+\frac{1}{2}}\left(\rho \partial_{x} \phi\right)_{j+\frac{1}{2}}\right)\right)
\end{array}\right.
$$

and $M_{j+\frac{1}{2}}=\frac{2 \rho c_{j+\frac{1}{2}} \varepsilon^{\beta}}{2 \rho c_{j+\frac{1}{2}} \varepsilon^{\beta}+\Delta x_{j+\frac{1}{2}} \sigma_{j+\frac{1}{2}} \rho_{j+\frac{1}{2}}}$.

These modifications allow us to correct the Lagrangian velocity and the numerical pressure viscosity to obtain the asymptotic preserving property. Indeed the coefficient $M$ modifies the numerical viscosity and when $\varepsilon$ is small the Lagrangian velocity gives the gradient of the pressure with the good diffusion coefficient (the numerical viscosity is not homogeneous to $\frac{\Delta x}{\varepsilon^{\alpha}}$ in this case). 
In the previous work we have noticed that to obtain an entropic scheme and to capture the steady states, it will important the modification of the fluxes is not compatible with the discretization of the source terms. For this reason, we propose to modify the latter to obtain the following scheme:

$$
\left\{\begin{array}{c}
\partial_{t} \rho_{j}+\frac{u_{j+\frac{1}{2}}^{*} \rho_{j+\frac{1}{2}}-u_{j-\frac{1}{2}}^{*} \rho_{j-\frac{1}{2}}}{\varepsilon^{\alpha} \Delta x_{j}}=0 \\
\partial_{t}(\rho u)_{j}+\frac{u_{j+\frac{1}{2}}^{*}(\rho u)_{j+\frac{1}{2}}-u_{j-\frac{1}{2}}^{*}(\rho u)_{j-\frac{1}{2}}}{\varepsilon^{\alpha} \Delta x_{j}}+\frac{p_{j+\frac{1}{2}}-p_{j-\frac{1}{2}}}{\varepsilon^{\alpha} \Delta x_{j}}= \\
-\frac{1}{2 \varepsilon^{\alpha}}\left(\left(\rho \partial_{x} \phi\right)_{j+\frac{1}{2}}+\frac{\sigma_{j+\frac{1}{2}}}{\varepsilon^{\beta}} \rho_{j+\frac{1}{2}} u_{j+\frac{1}{2}}^{*}+\left(\rho \partial_{x} \phi\right)_{j-\frac{1}{2}}+\frac{\sigma_{j-\frac{1}{2}}}{\varepsilon^{\beta}} \rho_{j-\frac{1}{2}} u_{j-\frac{1}{2}}^{*}\right) \\
\partial_{t}(\rho e)_{j}+\frac{u_{j+\frac{1}{2}}^{*}(\rho e)_{j+\frac{1}{2}}-u_{j-\frac{1}{2}}^{*}(\rho e)_{j-\frac{1}{2}}}{\varepsilon^{\alpha} \Delta x_{j}}+\frac{p_{j+\frac{1}{2}} u_{j+\frac{1}{2}}^{*}-p_{j-\frac{1}{2}} u_{j-\frac{1}{2}}^{*}}{\varepsilon^{\alpha} \Delta x_{j}}= \\
-\frac{1}{2 \varepsilon^{\alpha}}\left(u_{j+\frac{1}{2}}^{*}\left(\rho \partial_{x} \phi\right)_{j+\frac{1}{2}}+\frac{\sigma_{j+\frac{1}{2}}}{\varepsilon^{\beta}} \rho_{j+\frac{1}{2}} u_{j+\frac{1}{2}}^{*, 2}+u_{j-\frac{1}{2}}^{*}\left(\rho \partial_{x} \phi\right)_{j-\frac{1}{2}}+\frac{\sigma_{j-\frac{1}{2}}}{\varepsilon^{\beta}} \rho_{j-\frac{1}{2}} u_{j-\frac{1}{2}}^{*, 2}\right)
\end{array}\right.
$$

The scheme is given by (11)-(12). When we analyse the scheme obtained, we observe that it is very close to the Lagrange+remap scheme proposed in [9]. The main difference comes from to the original LR scheme. In [9] the authors use classical splitting Lagrange+remap scheme, whereas our scheme consists on: computing the Lagrangian phase, moving the mesh and, finally remapping the quantities on the original mesh. In our version, we write directly a Euler scheme without splitting. The second difference is that our scheme is written in non uniform grid to prepare for the $2 \mathrm{D}$ extension.

\subsection{Diffusion limit solutions: AP property}

In 4], for the linear case, we have shown that this type of discretization is not sufficient to obtain a scheme with a CFL independent of $\varepsilon$. To obtain this property, we propose to reformulate the scheme to obtain a local source term in the cell for the momentum equation. This formulation allows us to use a local implicit time scheme in the cell, which yields a stability independent of $\varepsilon$. We rewrite the scheme 12 , but splitting the pressure terms into two terms, each with an associated weight: $M_{j \pm \frac{1}{2}}$ and $1-M_{j \pm \frac{1}{2}}$. Firstly, we define

$$
A_{j+\frac{1}{2}}=-\frac{\left(1-M_{j+\frac{1}{2}}\right) p_{j+\frac{1}{2}}}{\varepsilon^{\alpha} \Delta x_{j}}-\frac{1}{2 \varepsilon^{\alpha}}\left(\rho_{j+\frac{1}{2}}\left(\partial_{x} \phi\right)_{j+\frac{1}{2}}+\frac{\sigma_{j+\frac{1}{2}}}{\varepsilon^{\beta}} \rho_{j+\frac{1}{2}} u_{j+\frac{1}{2}}^{*}\right)
$$

and $B_{j+\frac{1}{2}}=A_{j+\frac{1}{2}} u_{j+\frac{1}{2}}^{*}$. A quick computation shows that

$$
u_{j+\frac{1}{2}}^{*}=M_{j+\frac{1}{2}} u_{j+\frac{1}{2}}-M_{j+\frac{1}{2}} \frac{\Delta x_{j+\frac{1}{2}}}{2 \rho c_{j+\frac{1}{2}}}\left(\rho \partial_{x} \phi\right)_{j+\frac{1}{2}}
$$

with $u_{j+\frac{1}{2}}$ the classical Lagrangian velocity. Therefore, plugging 13 in the last term of $A_{j+\frac{1}{2}}$ and using $\frac{M_{j+\frac{1}{2}} \sigma_{j+\frac{1}{2}} \rho_{j+\frac{1}{2}}}{2 \varepsilon^{\beta}}=\frac{\rho c_{j+\frac{1}{2}}}{\Delta x_{j+\frac{1}{2}}}\left(1-M_{j+\frac{1}{2}}\right)$ for the gravity term, we obtain

$$
A_{j+\frac{1}{2}}=-\frac{\left(1-M_{j+\frac{1}{2}}\right) p_{j+\frac{1}{2}}}{\varepsilon^{\alpha} \Delta x_{j}}-\frac{1}{2 \varepsilon^{\alpha}} M_{j+\frac{1}{2}} \frac{\Delta x_{j+\frac{1}{2}}}{\Delta x_{j}}\left(\rho \partial_{x} \phi\right)_{j+\frac{1}{2}}-\frac{\rho c_{j+\frac{1}{2}}}{\varepsilon^{\alpha} \Delta x_{j+\frac{1}{2}}}\left(1-M_{j+\frac{1}{2}}\right) u_{j+\frac{1}{2}}^{*} \frac{\Delta x_{j+\frac{1}{2}}}{\Delta x_{j}}
$$

Thus, we have

$$
\left\{\begin{array}{l}
A_{j+\frac{1}{2}}=-\frac{\left(1-M_{j+\frac{1}{2}}\right) p_{j+\frac{1}{2}}+\rho c_{j+\frac{1}{2}}\left(1-M_{j+\frac{1}{2}}\right) u_{j+\frac{1}{2}}}{\varepsilon^{\alpha} \Delta x_{j}}-\frac{1}{2 \varepsilon^{\alpha}} M_{j+\frac{1}{2}} \frac{\Delta x_{j+\frac{1}{2}}}{\Delta x_{j}}\left(\rho \partial_{x} \phi\right)_{j+\frac{1}{2}} \\
A_{j-\frac{1}{2}}=\frac{\left(1-M_{j-\frac{1}{2}}\right) p_{j-\frac{1}{2}}-\rho c_{j-\frac{1}{2}}\left(1-M_{j-\frac{1}{2}}\right) u_{j-\frac{1}{2}}}{\varepsilon^{\alpha} \Delta x_{j}}-\frac{1}{2 \varepsilon^{\alpha}} M_{j-\frac{1}{2}} \frac{\Delta x_{j-\frac{1}{2}}}{\Delta x_{j}}\left(\rho \partial_{x} \phi\right)_{j-\frac{1}{2}}
\end{array}\right.
$$


Using the definitions of the fluxes $p_{j+\frac{1}{2}}^{*}$ and $u_{j+\frac{1}{2}}$, it yields

$$
\begin{aligned}
A_{j+\frac{1}{2}}-A_{j+\frac{1}{2}}= & \frac{M_{j+\frac{1}{2}}-M_{j-\frac{1}{2}}}{\varepsilon^{\alpha} \Delta x_{j}} p_{j}-\frac{1}{2 \varepsilon^{\alpha}}\left(M_{j+\frac{1}{2}}\left(\rho \partial_{x} \phi\right)_{j+\frac{1}{2}}+M_{j-\frac{1}{2}}\left(\rho \partial_{x} \phi\right)_{j-\frac{1}{2}}\right) \\
& -\left(\rho_{j+\frac{1}{2}} M_{j+\frac{1}{2}} \frac{\sigma_{j+\frac{1}{2}}}{2 \varepsilon^{\gamma}}+\rho_{j-\frac{1}{2}} M_{j-\frac{1}{2}} \frac{\sigma_{j-\frac{1}{2}}}{2 \varepsilon^{\gamma}}\right) u_{j}
\end{aligned}
$$

and $B_{j+\frac{1}{2}}-B_{j-\frac{1}{2}}=A_{j+\frac{1}{2}} u_{j-\frac{1}{2}}^{*}-A_{j+\frac{1}{2}} u_{j+\frac{1}{2}}^{*}(\gamma=\alpha+\beta)$. At the end, we get the following scheme

$$
\left\{\begin{array}{l}
\partial_{t} \rho_{j}+\frac{u_{j+\frac{1}{2}}^{*} \rho_{j+\frac{1}{2}}-u_{j-\frac{1}{2}}^{*} \rho_{j-\frac{1}{2}}}{\varepsilon^{\alpha} \Delta x_{j}}=0 \\
\partial_{t}(\rho u)_{j}+\frac{u_{j+\frac{1}{2}}^{*}(\rho u)_{j+\frac{1}{2}}-u_{j-\frac{1}{2}}^{*}(\rho u)_{j-\frac{1}{2}}}{\varepsilon^{\alpha} \Delta x_{j}}+\frac{p_{j+\frac{1}{2}}^{*}-p_{j-\frac{1}{2}}^{*}}{\varepsilon^{\alpha} \Delta x_{j}}=\frac{M_{j+\frac{1}{2}}-M_{j-\frac{1}{2}}}{\varepsilon^{\alpha} \Delta x} p_{j} \\
-\frac{1}{2 \varepsilon^{\alpha}}\left(\frac{\Delta x_{j+\frac{1}{2}}}{\Delta x_{j}} M_{j+\frac{1}{2}}\left(\left(\rho \partial_{x} \phi\right)_{j+\frac{1}{2}}+\rho_{j+\frac{1}{2}} \frac{\sigma_{j+\frac{1}{2}}}{\varepsilon^{\beta}} u_{j}\right)+\frac{\Delta x_{j-\frac{1}{2}}}{\Delta x_{j}} M_{j-\frac{1}{2}}\left(\left(\rho \partial_{x} \phi\right)_{j-\frac{1}{2}}+\rho_{j-\frac{1}{2}} \frac{\sigma_{j-\frac{1}{2}}}{\varepsilon^{\beta}} u_{j}\right)\right) \\
\partial_{t}(\rho e)_{j}+\frac{u_{j+\frac{1}{2}}^{*}(\rho e)_{j+\frac{1}{2}}-u_{j-\frac{1}{2}}^{*}(\rho e)_{j-\frac{1}{2}}}{\varepsilon^{\alpha} \Delta x_{j}}+\frac{p_{j+\frac{1}{2}}^{*} u_{j+\frac{1}{2}}^{*}-p_{j-\frac{1}{2}}^{*} u_{j-\frac{1}{2}}^{*}}{\varepsilon^{\alpha} \Delta x_{j}}=-\frac{\left(1-M_{j+\frac{1}{2}}\right) u_{j+\frac{1}{2}}^{*}-\left(1-M_{j-\frac{1}{2}}\right) u_{j-\frac{1}{2}}^{*}}{\varepsilon^{\alpha} \Delta x} p_{j} \\
-\frac{1}{2 \varepsilon^{\alpha}}\left(\frac{\Delta x_{j+\frac{1}{2}}}{\Delta x_{j}} M_{j+\frac{1}{2}} u_{j+\frac{1}{2}}^{*}\left(\left(\rho \partial_{x} \phi\right)_{j+\frac{1}{2}}+\rho_{j+\frac{1}{2}} \frac{\sigma_{j+\frac{1}{2}}}{\varepsilon^{\beta}} u_{j}\right)+\frac{\Delta x_{j-\frac{1}{2}}}{\Delta x_{j}} M_{j-\frac{1}{2}} u_{j-\frac{1}{2}}^{*}\left(\left(\rho \partial_{x} \phi\right)_{j-\frac{1}{2}}+\rho_{j-\frac{1}{2}} \frac{\sigma_{j-\frac{1}{2}}}{\varepsilon^{\beta}} u_{j}\right)\right)
\end{array}\right.
$$

with the modified Lagrangian fluxes

$$
\left\{\begin{array}{l}
p_{j+\frac{1}{2}}^{*}=M_{j+\frac{1}{2}}\left(\frac{1}{2}\left(p_{j}+p_{j+1}\right)+\frac{1}{2} \rho c_{j+\frac{1}{2}}\left(u_{j}-u_{j+1}\right)\right) \\
u_{j+\frac{1}{2}}^{*}=M_{j+\frac{1}{2}}\left(\frac{1}{2}\left(u_{j}+u_{j+1}\right)+\frac{1}{2 \rho c_{j+\frac{1}{2}}}\left(p_{j}-p_{j+1}-\Delta x_{j+\frac{1}{2}}\left(\rho \partial_{x} \phi\right)_{j+\frac{1}{2}}\right)\right.
\end{array}\right.
$$

Since $M=O\left(\varepsilon^{\beta}\right)$, we have just one stiff term. This term is the term of friction for the momentum equation. With this new formulation we can write a local implicit friction source term in the cell, substituting $u_{j}$ by $\frac{\left(u_{j}\right)^{n+1}}{\rho_{j}^{n+1}}$ in the momentum equation. Using a implicit time scheme for this term we can obtain a CFL independent of $\varepsilon$. This property will be verify only numerically in this paper. The final scheme is given by (14) - 15. Let us prove formally that the scheme is asymptotic preserving. When we compute formally the limit of our scheme we obtain a diffusion scheme with the good diffusion coefficient consistant with the limit diffusion equation.

Lemma 2.1. We suppose that the discrete density and internal energy are strictly positive. We assume that $\alpha=\beta=1$. When $\varepsilon$ tends to zero, the scheme (14) - 15) tends to

$$
\left\{\begin{array}{l}
\partial_{t} \rho_{j}^{0}+\frac{u_{j+\frac{1}{2}}^{*} \rho_{j+\frac{1}{2}}-u_{j-\frac{1}{2}}^{*} \rho_{j-\frac{1}{2}}}{\Delta x_{j}}=0 \\
\partial_{t}(\rho e)_{j}+\frac{u_{j+\frac{1}{2}}^{*}(\rho e)_{j+\frac{1}{2}}-u_{j-\frac{1}{2}}^{*}(\rho e)_{j-\frac{1}{2}}}{\Delta x_{j}}+\frac{u_{j+\frac{1}{2}}^{*}-u_{j-\frac{1}{2}}^{*}}{\Delta x_{j}} p_{j}=0
\end{array}\right.
$$

with $u_{j+\frac{1}{2}}^{*}=-\frac{1}{\sigma_{j+\frac{1}{2}}}\left(\frac{p_{j+1}-p_{j}}{\rho_{j+\frac{1}{2}} \Delta x_{j+\frac{1}{2}}}+\left(\partial_{x} \phi\right)_{j+\frac{1}{2}}\right)$. 
Proof. We begin by defining $M_{j+\frac{1}{2}}^{*}=\frac{2 \rho c_{j+\frac{1}{2}}}{2 \rho c_{j+\frac{1}{2}} \varepsilon^{\beta}+\sigma_{j+\frac{1}{2}} \rho_{j+\frac{1}{2}} \Delta x_{j+\frac{1}{2}}}=\frac{M_{j+\frac{1}{2}}}{\varepsilon^{\beta}}$. When introducing these new coefficients in the scheme and in the fluxes, it follows that

$$
\left\{\begin{array}{l}
p_{j+\frac{1}{2}}^{*}=M_{j+\frac{1}{2}}^{*}\left(\frac{1}{2}\left(p_{j}+p_{j+1}\right)+\frac{1}{2} \rho c_{j+\frac{1}{2}}\left(u_{j}-u_{j+1}\right)\right) \\
u_{j+\frac{1}{2}}^{*}=M_{j+\frac{1}{2}}^{*}\left(\frac{1}{2}\left(u_{j}+u_{j+1}\right)+\frac{1}{2 \rho c_{j+\frac{1}{2}}}\left(p_{j}-p_{j+1}-\Delta x_{j+\frac{1}{2}}\left(\rho \partial_{x} \phi\right)_{j+\frac{1}{2}}\right)\right)
\end{array}\right.
$$

When $\varepsilon$ tend to zero, the coefficient $M_{j+\frac{1}{2}}^{*} \approx \frac{2 \rho c_{j+\frac{1}{2}}}{\sigma_{j+\frac{1}{2}} \rho_{j+\frac{1}{2}} \Delta x_{j+\frac{1}{2}}}$.

Now we use a Hilbert expansion. For each variable we use a expansion like $\rho_{j}=\rho_{j}^{0}+\varepsilon \rho_{j}^{1}+\varepsilon^{2} \rho_{j}^{2}+$ $o\left(\varepsilon^{2}\right)$.

$\operatorname{Term} \frac{1}{\varepsilon}:\left(\rho_{j+\frac{1}{2}}^{0} M_{j+\frac{1}{2}}^{*} \sigma_{j+\frac{1}{2}}+\rho_{j-\frac{1}{2}}^{0} M_{j-\frac{1}{2}}^{*} \sigma_{j-\frac{1}{2}}\right) u_{j}^{0}=0$, consequently $u_{j}=0^{0}$.

Term O(1):

$$
\begin{aligned}
& \left\{\begin{array}{l}
p_{j+\frac{1}{2}}^{*}=M_{j+\frac{1}{2}}^{*}\left(\frac{1}{2}\left(p_{j}^{0}+p_{j+1}^{0}\right)\right) \\
u_{j+\frac{1}{2}}^{*}=-\left(\frac{p_{j+1}^{0}-p_{j}^{0}}{\sigma_{j+\frac{1}{2}} \rho_{j+\frac{1}{2}}^{0} \Delta x_{j+\frac{1}{2}}}+\frac{\left(\rho \partial_{x} \phi\right)_{j+\frac{1}{2}}}{\rho_{j+\frac{1}{2}} \sigma_{j+\frac{1}{2}}}\right)
\end{array}\right. \\
& \left\{\begin{array}{l}
\partial_{t} \rho_{j}^{0}+\frac{u_{j+\frac{1}{2}}^{*} \rho_{j+\frac{1}{2}}^{0}-u_{j-\frac{1}{2}}^{*} \rho_{j-\frac{1}{2}}^{0}}{\Delta x_{j}}=0 \\
\frac{p_{j+\frac{1}{2}}^{*}-p_{j-\frac{1}{2}}^{*}}{\Delta x}-\frac{M_{j+\frac{1}{2}}^{*}-M_{j-\frac{1}{2}}^{*}}{\Delta x_{j}} p_{j}= \\
-\frac{1}{2}\left(\frac{\Delta x_{j+\frac{1}{2}}}{\Delta x_{j}} M_{j+\frac{1}{2}}^{*}\left(\left(\rho \partial_{x} \phi\right)_{j+\frac{1}{2}}^{0}+\rho_{j+\frac{1}{2}} \sigma_{j+\frac{1}{2}} u_{j}^{1}\right)+\frac{\Delta x_{j-\frac{1}{2}}}{\Delta x_{j}} M_{j-\frac{1}{2}}^{*}\left(\left(\rho \partial_{x} \phi\right)_{j-\frac{1}{2}}^{0}+\rho_{j-\frac{1}{2}} \sigma_{j-\frac{1}{2}} u_{j}^{1}\right)\right. \\
\partial_{t}(\rho e)_{j}+\frac{u_{j+\frac{1}{2}}^{*}(\rho e)_{j+\frac{1}{2}}^{0}-u_{j-\frac{1}{2}}^{*}(\rho e)_{j-\frac{1}{2}}^{0}}{\Delta x_{j}}+\frac{u_{j+\frac{1}{2}}^{*}-u_{j-\frac{1}{2}}^{*}}{\Delta x_{j}} p_{j}=0
\end{array}\right.
\end{aligned}
$$

This formula shows that at the limit, we get a scheme with the good diffusion coefficient and a gradient at the interface consistant on non uniform grid (if we apply this formal expansion to the classical scheme we obtain a diffusion coefficient which depend of $\varepsilon$ and a gradient at the interface not consistant). Consequently, formally the scheme capture correctly (error independent of $\varepsilon$ ) the diffusion solutions (3). In the literature of asymptotic preserving scheme, this is known as the "magic" coefficient $M_{j \pm \frac{1}{2}}$ which allows us to modify and to correct the diffusion coefficient and the pressure gradient at the interface.

\subsection{Steady solutions: Well-Balanced property}

When we use a classical Godunov type scheme to treat the steady states like $\partial_{x} p=-\rho \partial_{x} \phi$ and $u=0$ obtain with $\alpha=1$ and $\beta=0$ and also the steady solutions (4) for $\beta=1$ and $\alpha$, some spurious numerical velocities homogeneous to $O(\Delta x)$ appear. For nearly uniform flows (example: atmospheric flow) this numerical artefacts are larger that physical velocities and can destroy the accuracy of the simulation. Consequently it is important to design a numerical method adapted to treat steady states. In this section we will discuss the steady solutions (5) associated to the long 
time behavior. Indeed the steady solutions associated to the subset of solutions 4 is more simple ti study. Study the solutions we remark that the velocity is homogeneous to $O\left(\varepsilon^{\beta}\right)$. Study the fluxes we remark that the numerical velocity at the interface is homogeneous to $O\left(\Delta x\left\|\partial_{x} p\right\|\right)$. For the new scheme called LR-AP scheme $\sqrt{14}$, the presence of the coefficient $M$ allows to obtain a numerical velocity at the interface to $O\left(\varepsilon^{\beta}\left\|\partial_{x} p\right\|\right)$. We will validate this remark in the numerical section.

Now we study the steady states given by (5) called hydrostatic equilibrium. For this the wellbalanced property as a scheme which discretize with a good accuracy the steady states. This type of scheme allows us to reduce the spurious velocities. For equations with algebraic steady state like Shallow-Water equations the well balanced scheme discretize exactly the equilibrium. For Euler equations with gravity the hydrostatic equilibriums are differentials. This makes it difficult to capture all this subset of solutions.

Definition 2.1 (Well-balanced property). A scheme is well-balanced if the scheme is exact for a initial data given by the discrete steady states at the interfaces. For the Euler equation with gravity the discrete steady state is $\left(\partial_{x} p\right)_{j+\frac{1}{2}}=-\left(\rho \partial_{x} \phi\right)_{j+\frac{1}{2}}$ with $\left(\partial_{x} p\right)_{j+\frac{1}{2}}$ a discrete gradient at the interface and $\left(\rho \partial_{x} \phi\right)_{j+\frac{1}{2}}$ a discretization of $\rho \partial_{x} \phi$ at the interface.

With this definition we notice that the order of the error in space for the steady state is only governed by the numerical error between the discrete steady state and the continuous steady state. Indeed, if the initial error between the continuous steady state and discrete steady state is homogeneous to $O\left(\Delta x^{p}\right)$, the spatial error after the time would be of the same order. For example, we have the scheme

$$
\mathbf{V}^{n+1}=\mathbf{V}^{n}-\Delta t F\left(\mathbf{V}^{n}\right)
$$

We take $\mathbf{V}^{*}$ with $F\left(\mathbf{V}^{*}\right)=0$ and $\mathbf{V}^{n}=\mathbf{V}^{*}+\delta \mathbf{V}$. In this case

$$
\mathbf{V}^{n+1}=\mathbf{V}^{n}-\Delta t F\left(\mathbf{V}^{n}\right)=\mathbf{V}^{n}-\Delta t\left(F\left(\mathbf{V}^{*}\right)+\delta \mathbf{V}\left(F^{\prime}\left(\mathbf{V}^{*}\right)\right)\right)=\mathbf{V}^{*}+\left[I_{d}-\Delta t F^{\prime}\left(\mathbf{V}^{*}\right)\right] \delta \mathbf{V}
$$

If $\delta \mathbf{V}=O\left(\Delta x^{p}\right)$ and assuming that the scheme is stable $\left(\max _{\mathbf{V}}\left\|I_{d}-\Delta t F^{\prime}(\mathbf{V})\right\|<1\right)$, the error is homogeneous to $O\left(\Delta x^{p}\right)$. To obtain a good accuracy it is sufficient to work only on the discrete steady state. This reduction of the problem allows us to design easily some numerical methods.

Proposition 2.2. We choose the explicit discretization of the scheme 14 15). We assume that the initial data satisfies $\left(\partial_{x} p\right)_{j+\frac{1}{2}}=-\left(\rho \partial_{x} \phi\right)_{j+\frac{1}{2}}$ which is equivalent to $p_{j+1}-p_{j}=-\Delta x_{j+\frac{1}{2}}\left(\rho \partial_{x} \phi\right)_{j+\frac{1}{2}}$ and $u_{j}=0$ then $\rho_{j}^{n+1}=\rho_{j}, u_{j}^{n+1}=0$ and $(\rho e)_{j}^{n+1}=\rho_{j} e_{j}$.

Proof. We begin by studying the following solver

$$
u_{j+\frac{1}{2}}^{*}=M_{j+\frac{1}{2}}\left(\frac{1}{2}\left(u_{j}+u_{j+1}\right)+\frac{1}{2 \rho c_{j+\frac{1}{2}}}\left(p_{j}-p_{j+1}-\Delta x_{j+\frac{1}{2}}\left(\rho \partial_{x} \phi\right)_{j+\frac{1}{2}}\right)\right)
$$

since $u_{j}=0$ and $\frac{p_{j+1}-p_{j}}{\Delta x_{j+\frac{1}{2}}}=-\left(\rho \partial_{x} \phi\right)_{j+\frac{1}{2}}$ it follows that

$$
u_{j+\frac{1}{2}}^{*}=M_{j+\frac{1}{2}} \frac{1}{2 \rho c_{j+\frac{1}{2}}}\left(\left(\rho \partial_{x} \phi\right)_{j+\frac{1}{2}} \Delta x_{j+\frac{1}{2}}-\Delta x_{j+\frac{1}{2}}\left(\rho \partial_{x} \phi\right)_{j+\frac{1}{2}}\right)=0
$$

and $\rho_{j}^{n+1}=\rho_{j}$, and $(\rho e)_{j}^{n+1}=\rho_{j} e_{j}$. To finish we notice that $\left.p_{j+\frac{1}{2}}^{*}=M_{j+\frac{1}{2}} \frac{1}{2}\left(p_{j}+p_{j+1}\right)\right)$, consequently

$$
\begin{aligned}
\frac{p_{j+\frac{1}{2}}^{*}-p_{j-\frac{1}{2}}^{*}}{2 \varepsilon^{\alpha} \Delta x_{j}}-\frac{M_{j+\frac{1}{2}}-M_{j-\frac{1}{2}}}{\varepsilon^{\alpha} \Delta x_{j}} p_{j} & =\frac{M_{j+\frac{1}{2}}\left(p_{j+1}-p_{j}\right)+M_{j-\frac{1}{2}}\left(p_{j}-p_{j-1}\right)}{\varepsilon^{\alpha} \Delta x_{j}} \\
& =-\frac{M_{j+\frac{1}{2}} \Delta x_{j+\frac{1}{2}}\left(\rho \partial_{x} \phi\right)_{j+\frac{1}{2}}+M_{j-\frac{1}{2}} \Delta x_{j-\frac{1}{2}}\left(\rho \partial_{x} \phi\right)_{j-\frac{1}{2}}}{2 \Delta x_{j} \varepsilon^{\alpha}}
\end{aligned}
$$

The previous term of the fluxes is equal to the gravity source term. Since the friction source term is equal to zero we obtain the result. 
For the hydrostatic steady states the numerical error associated with the LP-AP scheme 14 15 comes from only the consistency error between $\partial_{x} p=-\rho \partial_{x} \phi$ and $\left(\partial_{x} p\right)_{j+\frac{1}{2}}=-\left(\rho \partial_{x} \phi\right)_{j+\frac{1}{2}}$ with $\left(\partial_{x} p\right)_{j+\frac{1}{2}}=-\frac{p_{j+1}-p_{j}}{\Delta x_{j+\frac{1}{2}}}$. Therefore to reduce the error for the steady states or around the steady sates, we must reduce the error between the continuous steady states and the discrete steady state. To begin we consider the following simple steady state $\partial_{x} p=-\rho \partial_{x} \phi$. Integrating on the diamond cell $\left[x_{j}, x_{j+1}\right]$ it yields

$$
p\left(x_{j+1}\right)-p\left(x_{j}\right)=-\int_{x_{j}}^{x_{j+1}} \rho(x) \partial_{x} \phi(x) .
$$

The first possibility to approximate the previous integral is to use $\left(\rho \partial_{x} \phi\right)_{j+\frac{1}{2}}=-\Delta x_{j+\frac{1}{2}} \rho_{j+\frac{1}{2}}\left(\partial_{x} \phi\right)_{j+\frac{1}{2}}$ with $\left(\partial_{x} \phi\right)_{j+\frac{1}{2}}=\frac{\phi_{j+1}-\phi_{j}}{\Delta x_{j+\frac{1}{2}}}$ and $\rho_{j+\frac{1}{2}}$ an average around the interface. We propose to use different averages:

- (R1): $\rho_{j+\frac{1}{2}}=\rho_{j}$. In this case the scheme is exact for steady states with constant density and converges with first order for the other steady states.

- (R2): $\rho_{j+\frac{1}{2}}=\frac{1}{2}\left(\rho_{j}+\rho_{j+1}\right)$. In this case the scheme converges with second order for the steady states. The scheme is exact for linear density on a general grid if we initialize the scheme with punctual values and not average values.

- (R3): $\rho_{j+\frac{1}{2}}=\frac{\rho_{j+1}-\rho_{j}}{\ln \left(\rho_{j+1}\right)-\ln \left(\rho_{j}\right)}$. In this case the scheme converges with second order for the other steady states. The scheme is exact for exponential density on general grid if we initialize the scheme with punctual values and not average values.

In the best case we obtain a second order discretization of the steady states (we will show this in the section on the numerical results). Now we propose to modify the discrete steady state to obtain easily an arbitrary high order discretization of the hydrostatic equilibrium. This modification is just a modification of the source term and the numerical viscosity. For this reason, this high order representation of the equilibrium should not generate Gibbs phenomenon and we keep the properties of stability associated to the first order finite volume schemes. Integrating the steady state on the diamond cell $\left[x_{j}, x_{j+1}\right]$ it yields

$$
\Delta x_{j+\frac{1}{2}}\left(\frac{1}{\Delta x_{j+\frac{1}{2}}} \int_{x_{j}}^{x_{j+1}} \partial_{x} p(x)\right)=-\Delta x_{j+\frac{1}{2}}\left(\frac{1}{\Delta x_{j+\frac{1}{2}}} \int_{x_{j}}^{x_{j+1}} \rho(x) \partial_{x} \phi(x)\right)
$$

Now we introduce three polynomials

$$
\bar{\rho}_{j+\frac{1}{2}}(x)=\sum_{k=1}^{q} r_{k} x^{k}, \quad \bar{p}_{j+\frac{1}{2}}(x)=\sum_{k=1}^{q+1} p_{k} x^{k}, \quad \bar{\phi}_{j+\frac{1}{2}}(x)=\sum_{k=1}^{q+1} \phi_{k} x^{k}
$$

with $\int_{x_{l-\frac{1}{2}}}^{x_{l+\frac{1}{2}}} \bar{\rho}_{j+\frac{1}{2}}(x)=\Delta x_{l} \rho_{l}, \int_{x_{l-\frac{1}{2}}}^{x_{l+\frac{1}{2}}} \bar{p}_{j+\frac{1}{2}}(x)=\Delta x_{l} p_{l}$ and $\int_{x_{l-\frac{1}{2}}}^{x_{l+\frac{1}{2}}} \bar{\phi}_{j+\frac{1}{2}}(x)=\Delta x_{l} \phi_{l}$ with $l \in S(j)$ $(S(j)$ is a subset of cells around $j$ with $N$ the number of element). These definitions of the polynomials and the conditions on the polynomials give linear systems which define the polynomials coefficients. For example for $\bar{\rho}_{j+\frac{1}{2}}$ the coefficients $r_{k}$ are solutions of the $n \times n$ system $A \mathbf{R}=\mathbf{b}$ with

$$
A_{k, l}=\int_{x_{l-\frac{1}{2}}}^{x_{l+\frac{1}{2}}} x^{k}, \quad b_{l}=\Delta x_{l} \rho_{l} \text { and } \quad R_{k}=r_{k} .
$$

Using these polynomials leads to the new discrete steady states

$$
\Delta x_{j+\frac{1}{2}}\left(\frac{1}{\Delta x_{j+\frac{1}{2}}} \int_{x_{j}}^{x_{j+1}} \partial_{x} \bar{p}_{j+\frac{1}{2}}(x)\right)=-\Delta x_{j+\frac{1}{2}}\left(\frac{1}{\Delta x_{j+\frac{1}{2}}} \int_{x_{j}}^{x_{j+1}} \bar{\rho}_{j+\frac{1}{2}}(x) \partial_{x} \bar{\phi}_{j+\frac{1}{2}}(x)\right) .
$$


Now we must modify these steady states. Indeed to obtain a scheme which preserves the discrete steady state, it is necessary to have the numerical pressure viscosity in the discrete steady states. Indeed, we incorpore the discrete equilibrium into the fluxes combining the discrete gradient of the pressure (numerical viscosity in the Lagrangian flux $u_{j \pm \frac{1}{2}}$ ) and in the discrete steady states. At the end the q-th order discrete hydrostatic equilibrium is defined by

$$
p_{j+1}-p_{j}=-\Delta x_{j+\frac{1}{2}}\left(\rho \partial_{x} \phi\right)_{j+\frac{1}{2}}^{H O}
$$

with

$$
(\rho g)_{j+\frac{1}{2}}^{H O}=\left(\frac{1}{\Delta x_{j+\frac{1}{2}}}\left(\int_{x_{j}}^{x_{j+1}} \partial_{x} \bar{p}_{j+\frac{1}{2}}(x)\right)+\left(\frac{1}{\Delta x_{j+\frac{1}{2}}} \int_{x_{j}}^{x_{j+1}} \bar{\rho}_{j+\frac{1}{2}}(x) \partial_{x} \bar{\phi}_{j+\frac{1}{2}}(x)\right)-\frac{p_{j+1}-p_{j}}{\Delta x_{j+\frac{1}{2}}}\right)
$$

Using the new coefficient $\left(\rho \partial_{x} \phi\right)_{j+\frac{1}{2}}^{H O}$ in the fluxes and the source terms of the scheme $140-\sqrt{15}$ we obtain a scheme with high order reconstruction of the equilibrium.

\subsection{Numerical results for AP properties}

In this subsection we give some numerical results to validate the asymptotic preserving property of the scheme 14 - 15 .

\section{High opacity asymptotic limit}

With this first test case, we propose to validate the asymptotic preserving for the high opacity limit $(\alpha=0$ and $\beta=1$ ). For this we propose a Sod test case for $\varepsilon \geq 1$ and we will compare the classical LR scheme and the LR-AP scheme for different values of $\varepsilon$. The domain is $\Omega=[-1,1]$. The final time is $T_{f}=0.2$ and $\varepsilon=1$. The grid is a random grid. The initial data is given by

$$
\left\{\begin{array}{l}
\rho(t=0)=1, u(t=0)=0, p(t=0)=1, \text { for } x<0 \\
\rho(t=0)=0.125, u(t=0)=0, p(t=0)=0.1, \text { for } x>0
\end{array}\right.
$$

When $\sigma$ tends to the infinity the stiff relaxation source term force $u=0$ at the limit. Consequently the initial solution is a stationary solution when $\sigma$ tends to the infinity.

In (Fig 1), we compare the classical and AP scheme for $\varepsilon=1$. The two schemes give comparable results. When $\varepsilon$ are close to 1 the AP scheme is just the classical scheme where the numerical viscosity and the discretization of the source terms have been modified. Consequently, it is normal that the two schemes give close results.

In (Fig 2) - (Fig 3), we compare the classical and the AP scheme for small $\varepsilon$. The solutions for small $\varepsilon$ given by the LR-AP scheme is very close to the initial data (in red). Hence the LR-AP scheme captures correctly the stationary solution which is the asymptotic limit. This is not the case for the classical scheme. The velocity is homogeneous to $\frac{1}{\sigma}$ like for the LR-AP scheme. Yet, unlike the LR-AP scheme, the density pressure and energy move in time. This behavior comes from to the numerical viscosity of the Lagrangian velocity in the density and energy equations which generate an error homogeneous to $\Delta x$ contrary to the LR-AP scheme where this Lagrangian velocity is very close to zero. These results show that using an asymptotic preserving scheme allows us to obtain better numerical results.

\section{Diffusion asymptotic limit}

With this second test case, we propose to validate the asymptotic preserving property for the high opacity limit and for long time $(\alpha=1$ and $\div e b t a=1)$. We propose a Sod test case with $\sigma=1$ and $\varepsilon=0.005$ and we will compare the classical LR scheme and the LR-AP scheme for different meshes. The domain is $\Omega=[-1,1]$. The final time is $T_{f}=0.005$. The grid is uniform. The initial data is given by

$$
\left\{\begin{array}{l}
\rho(t=0)=1, u(t=0)=0, p(t=0)=1, \text { for } x<0 \\
\rho(t=0)=0.125, u(t=0)=0, p(t=0)=0.1, \text { for } x>0
\end{array}\right.
$$



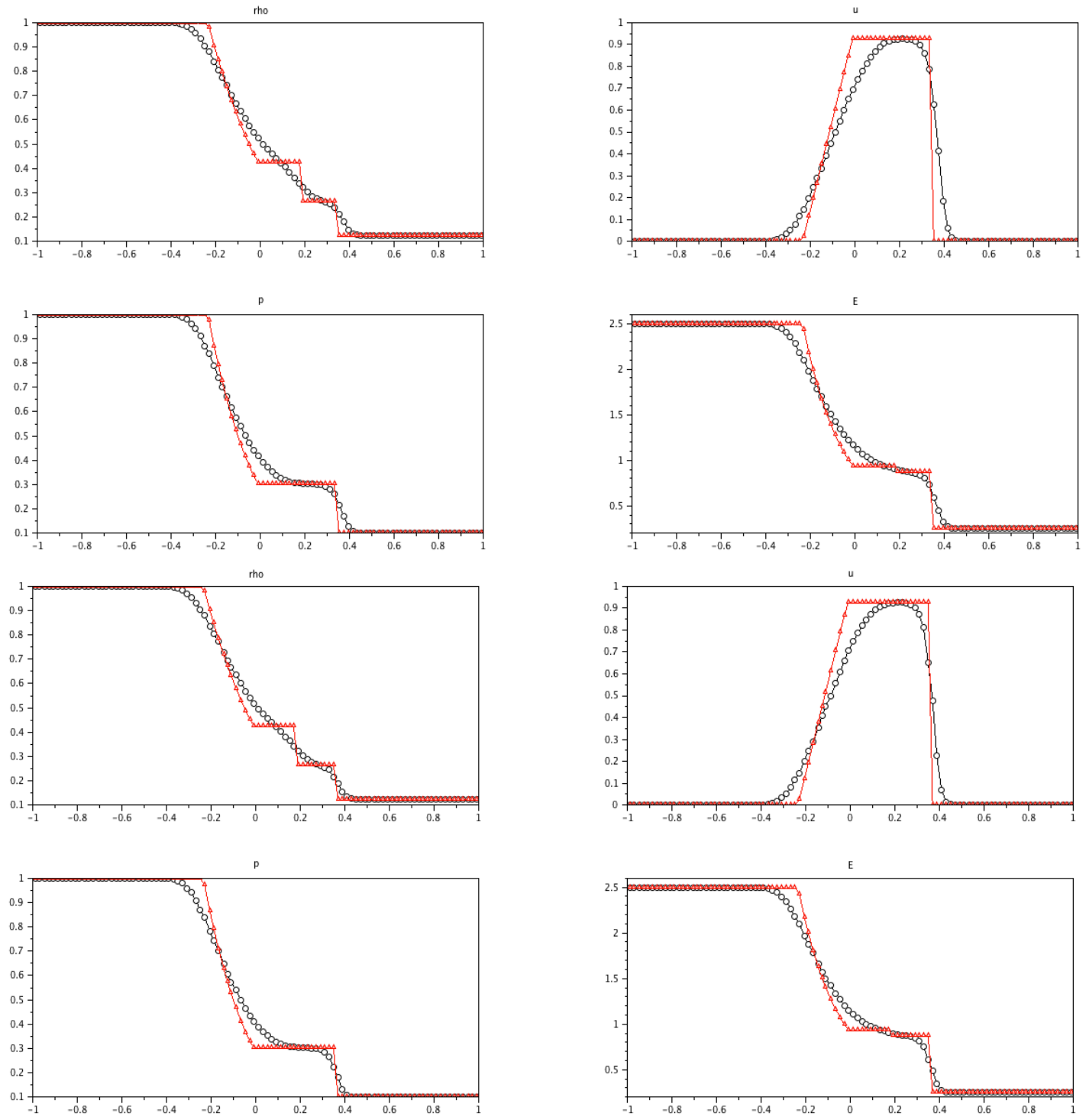

Figure 1: At the top we have the numerical solution (black) and the initial data (red) for the LR scheme and for $\sigma=1$. At the bottom we have the numerical solution (black) and the initial data (red) for the LR-AP scheme and for $\varepsilon=1$.

To validate the AP scheme, we propose to compare the solution given by the classical scheme on fine grid (5000 cells) and the solutions given by the two schemes on coarse grids (50 and 100 cells).

Comparing the Figure (Fig. 4) and the figures (Fig. 5) - (Fig. 6), we remark that the LR-AP scheme on coarse grids admit a time dynamic closer to the the time dynamic of the reference solution (reference solution computed on fine grid Fig .4) than the classical scheme. Indeed the time dynamic of the density and velocity profil given by the LR-AP are more close to the profiles given by the reference solution. The difference between the two schemes come from to the fact that the classical scheme admit a numerical viscosity homogeneous to $\frac{\Delta x}{\varepsilon}$ contrary to the LRAP scheme and will see on the figures that the classical scheme is more diffusive. The form of profiles between the reference solution and the solution given by the AP scheme is not so close (the reference solution is more smooth) but some computation for shorter time ( where the Cpu cost is 

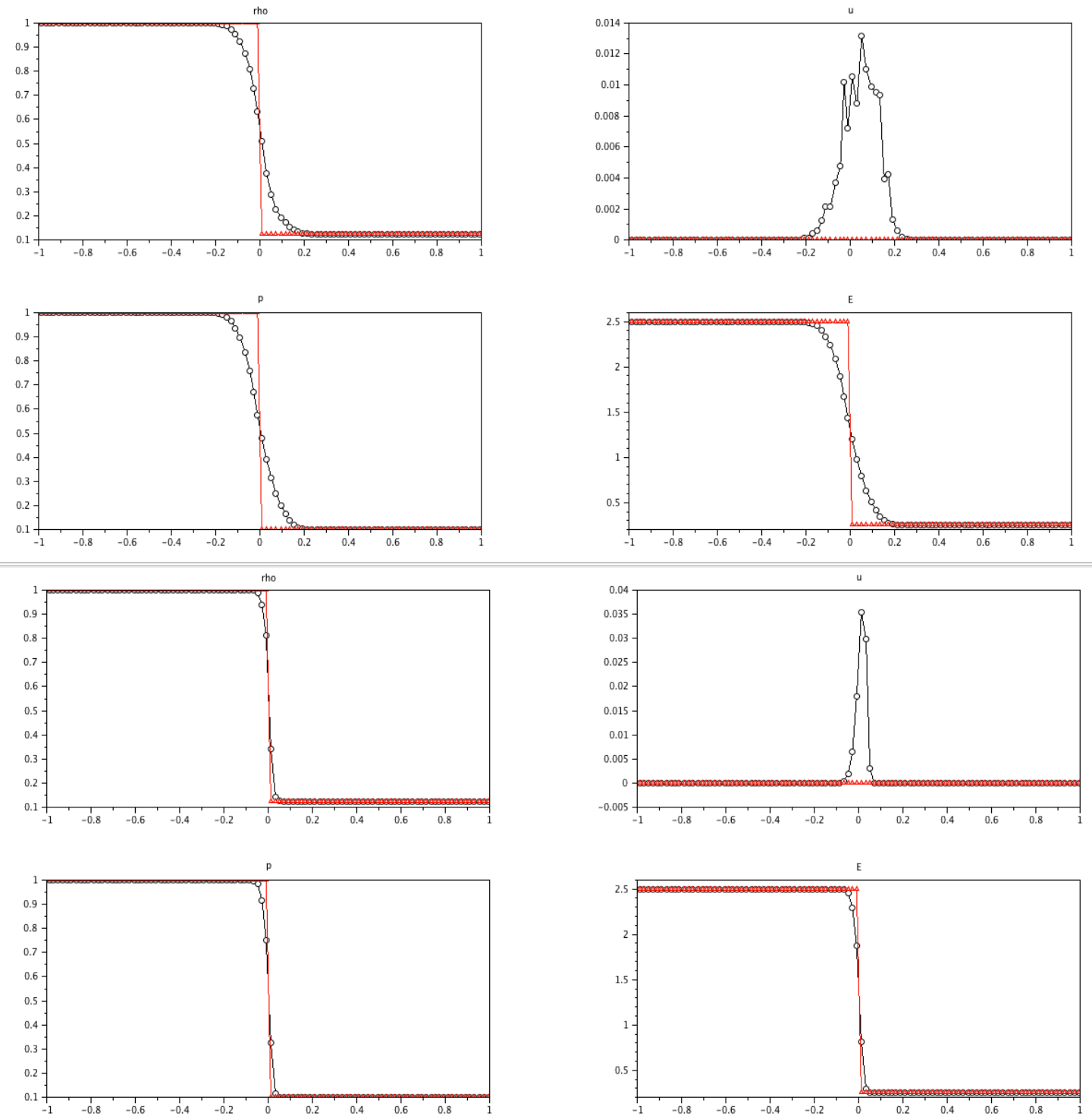

Figure 2: At the top we have the numerical solution (black) and the initial data (red) for the LR scheme and for $\varepsilon=1^{-3}$. At the bottom we have the numerical solution (black) and the initial data (red) for the LR-AP scheme and for $\varepsilon=1^{-3}$.

not too important) that the reference solution is closer to the AP solution when we decrease the step mesh.

\subsection{Numerical results for WB properties in 1D}

In this subsection we give some numerical results to show that the scheme (14) - 15 is wellbalanced and that it captures correctly the hydrostatic equilibrium 5 .

\section{Spatial convergence for steady states}

The LR AP scheme with $\rho_{j \pm \frac{1}{2}}$ and average (R1) is called LR-AP R1, the LR AP scheme with $\rho_{j \pm \frac{1}{2}}$ and average (R2) is is called LR-AP R2, and lastly, the LR AP scheme with $\rho_{j \pm \frac{1}{2}}$ and average 

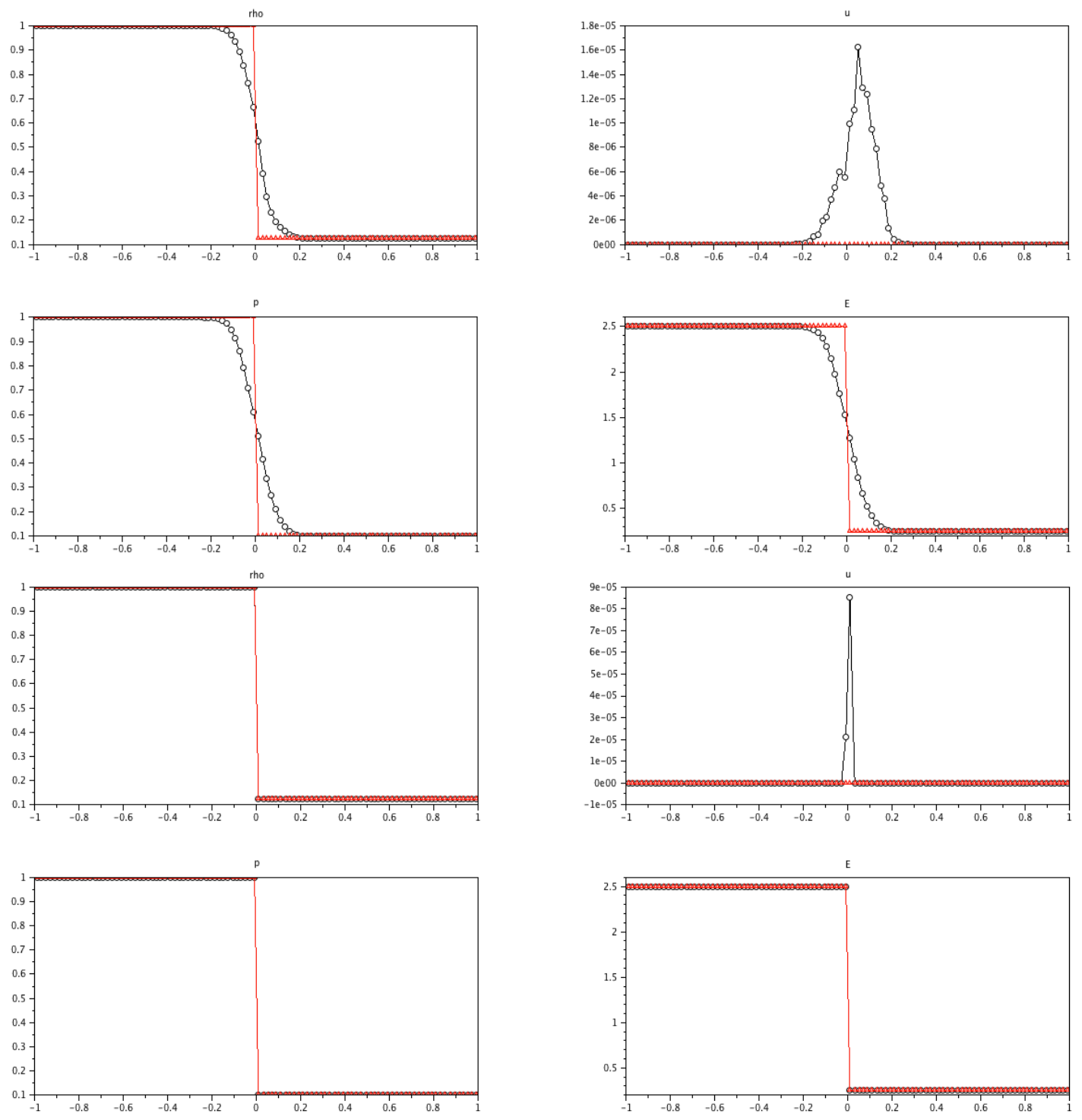

Figure 3: At the top we have the numerical solution (black) and the initial data (red) for the LR scheme and for $\varepsilon=10^{-6}$. At the bottom we have the numerical solution (black) and the initial data (red) for the LR-AP scheme and for $\varepsilon=10^{-6}$.

(R3) is is called LR-AP O(e). The LR AP scheme with $q$ high order discretization of the steady states is called LR-AP $O(q)$. We consider a domain $\Omega=[a, b]$. The difference test cases are given by

The scheme LR-AP $\mathrm{O}(2)$ which corresponds to the AP scheme with the mean (R2) for $\rho_{j+\frac{1}{2}}$ is exact for the linear density case only if we take the initial data with the punctual values of the solution. But for all the test cases we initialize the test cases with the average values. On non-uniform grids there is a difference between the punctual values and the averages values, for this reason the scheme LR-AP $\mathrm{O}(2)$ is not exact for the test case with linear density.

Some remarks about the results for the different steady states. Firstly the classical scheme converges for all test cases with order one. Secondly the scheme LR-AP (q) with q-th order approximation of the steady state (for variable or constant gravity) converges with the order $q$ as 

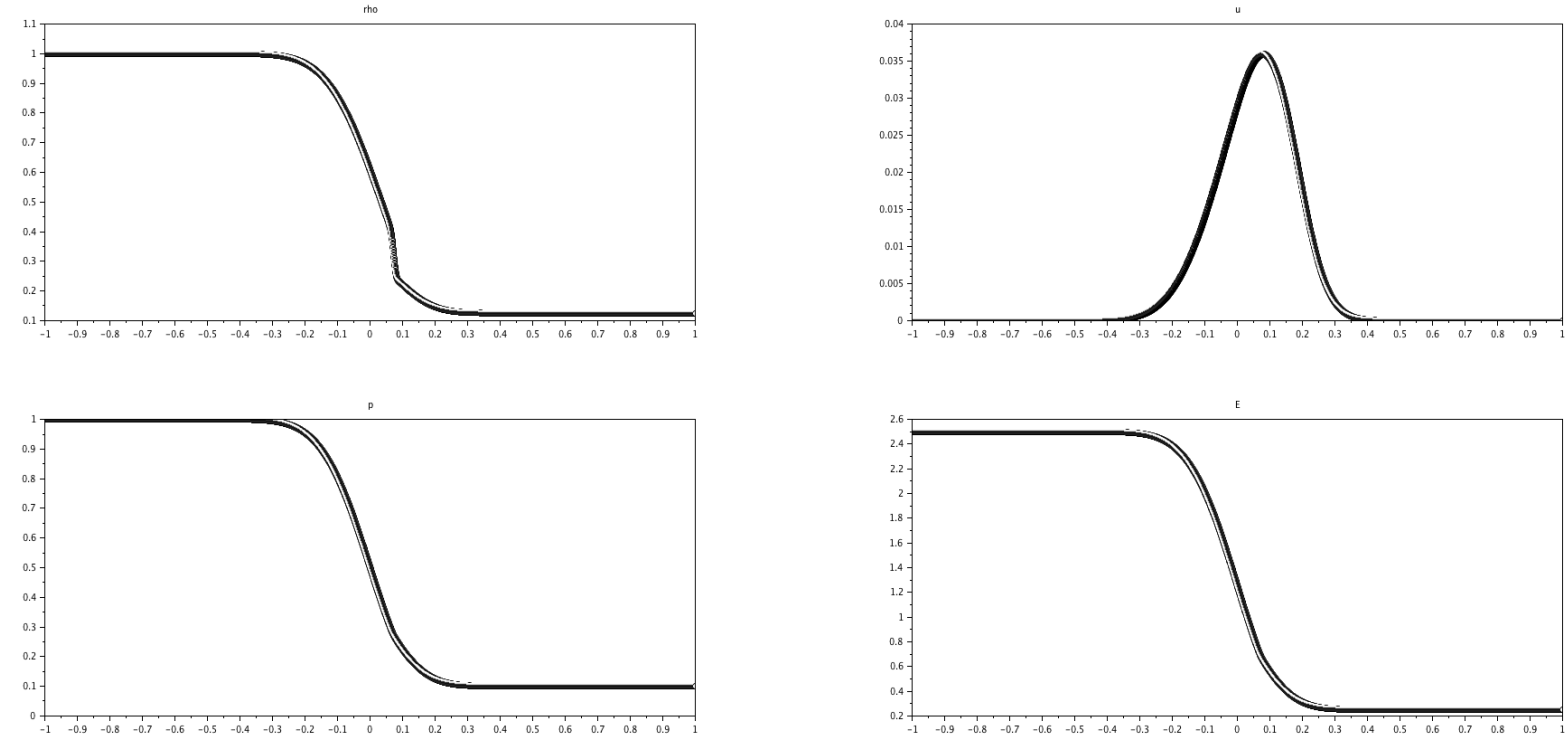

Figure 4: Solution given by the classical grid on fine grid (5000 cells).

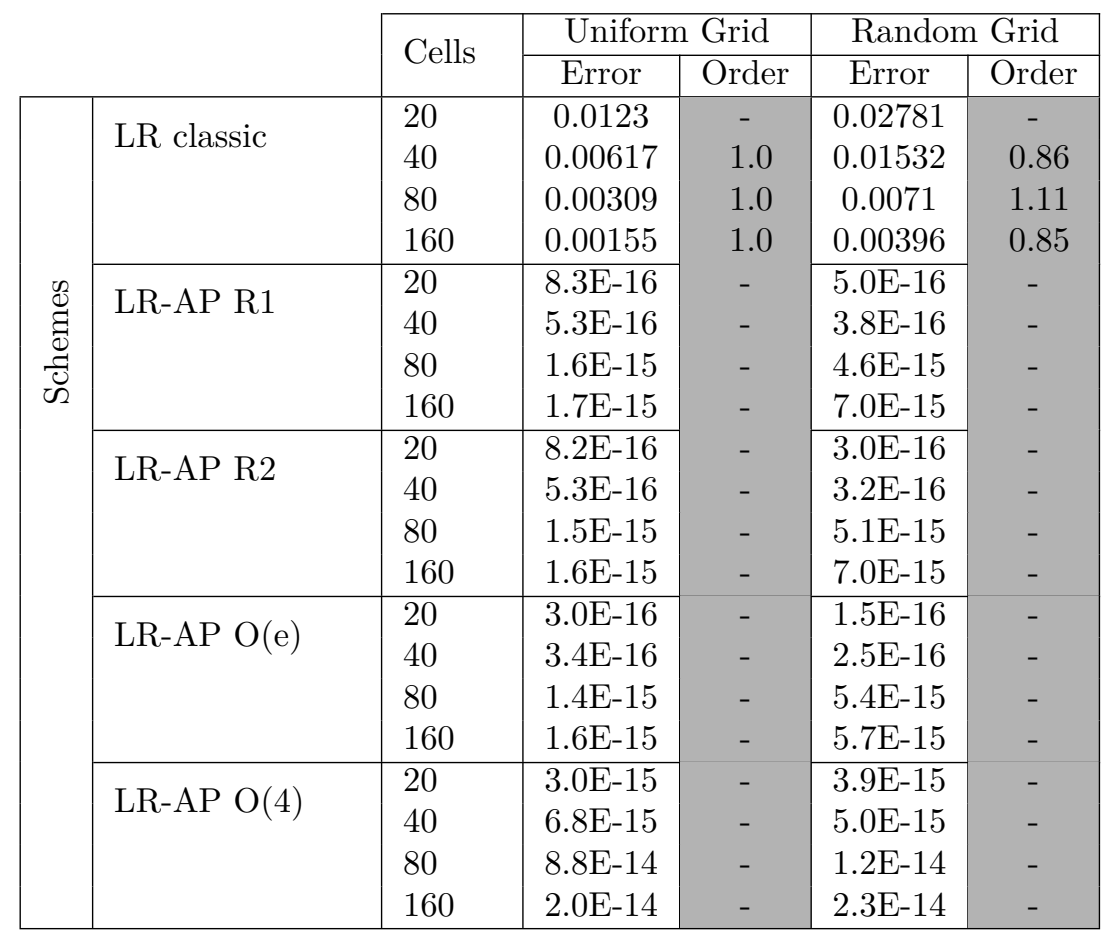

Table 1: Test case with constant density. Err corresponds to the $L 1$ error and $q$ corresponds to the order of convergence.

expected (the average $R 1$ corresponds to $q=1$, the average $R 2$ corresponds to $q=2$ ). If the density is a $q-1$ order polynomial the scheme is exact for the steady state, if the reconstruction is correctly designed. The average $R 2$ for example is exact for linear density on non-uniform grids if we use the punctual values. Although it converges with second order if we average values at the initialization of the scheme. Futhermore the scheme with average $R 3$ converges with the second 

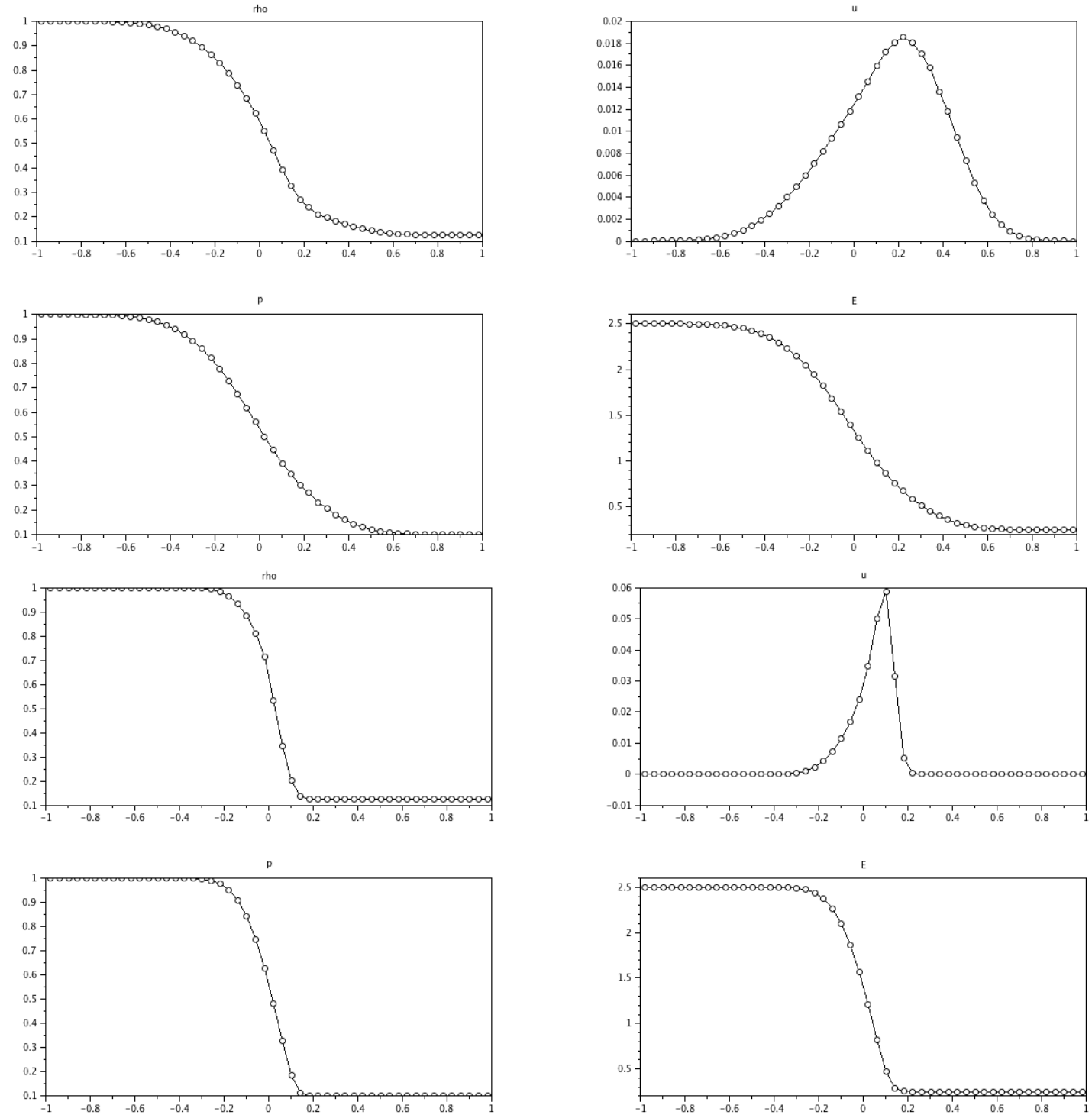

Figure 5: In top the solution given by the classical grid on coarse (50 cells). In bottom we have the solution given by the AP grid on coarse grid (50 cells).

order for the general case and is exact for the exponential case on uniform grids. At the end we observe that the final order of convergence is the same that the order of approximation used for the discrete steady states. Thus, as expected, we notice that the numerical convergence in space is only governed by the discrete steady states. 

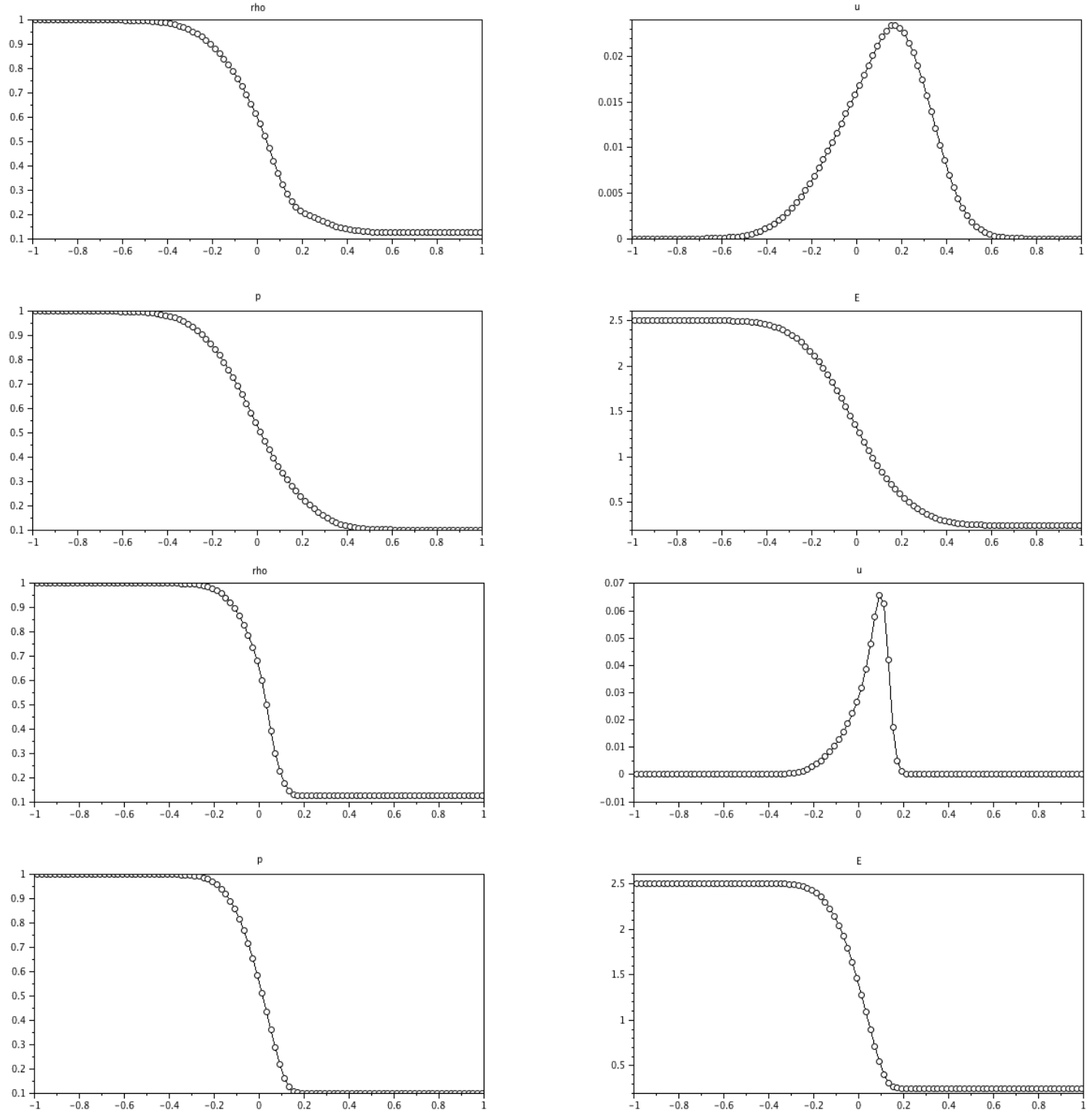

Figure 6: In top the solution given by the classical grid on coarse (100 cells). In bottom we have the solution given by the AP grid on coarse grid (100 cells).

\section{Extension on 2D unstructured meshes}

Now we propose to use the same technique coupled with finite volumes Nodal scheme to extend our method in 2D on unstructured meshes. Equivalently to the $1 \mathrm{D}$ case, we propose to begin with an one-step Lagrange+remap scheme (without splitting between Lagrangian and Remap steps). In [4] we have shown for the linear case that the nodal methods, where the fluxes are localized at the corner, in contrary to the classical schemes, are more adapted to design consistent asymptotic preserving scheme on unstructured meshes. Indeed the limit diffusion associated with the classical schemes (fluxes localized at the edge) is not consistant where the mesh does not satisfies the Delaunay conditions. With the nodal method, we have a limit diffusion scheme convergent on very distorted meshes. Consequently for the Euler equations we propose to begin with the Lagrange+remap nodal scheme. An extension have been introduced quickly in [12]. Now we propose to describe the construction of this extension and the properties of the method. 


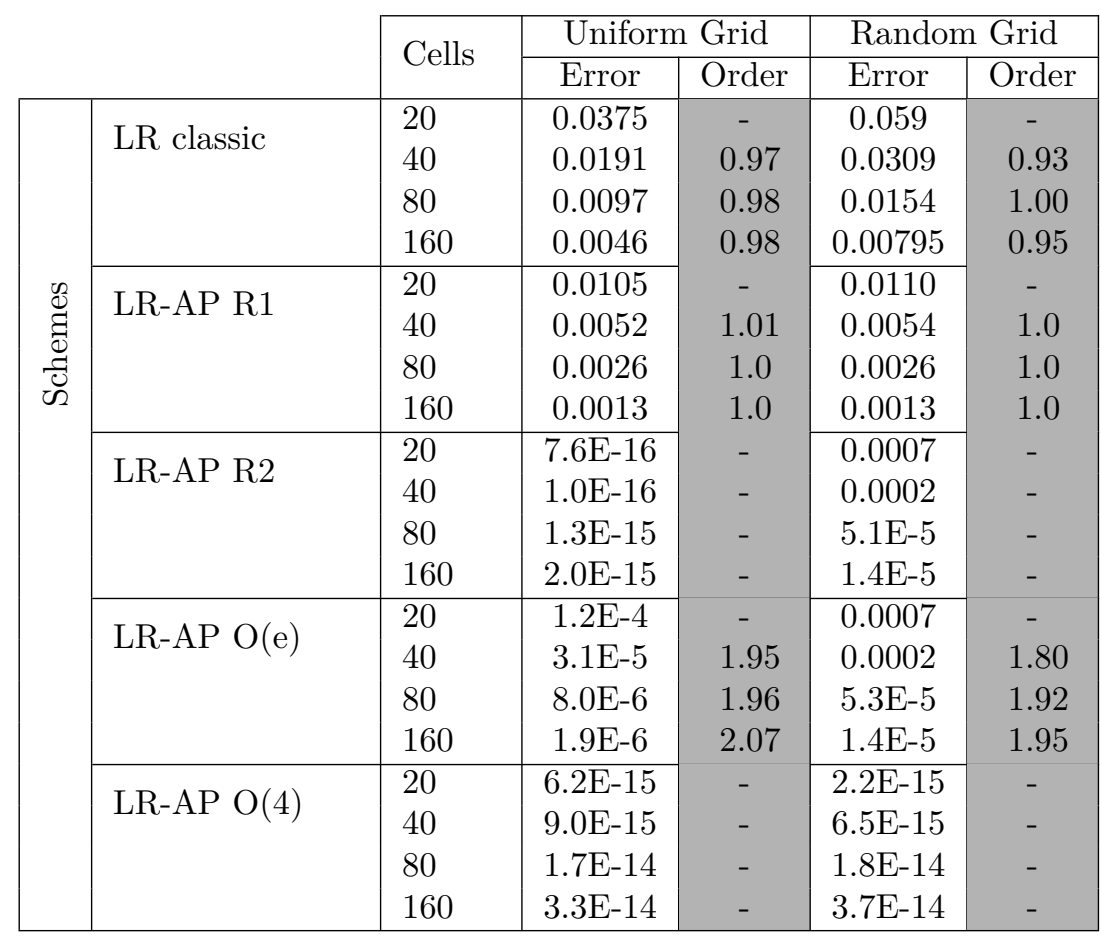

Table 2: Test case with linear density. Err corresponds to the $L 1$ error and $q$ to the order of convergence.

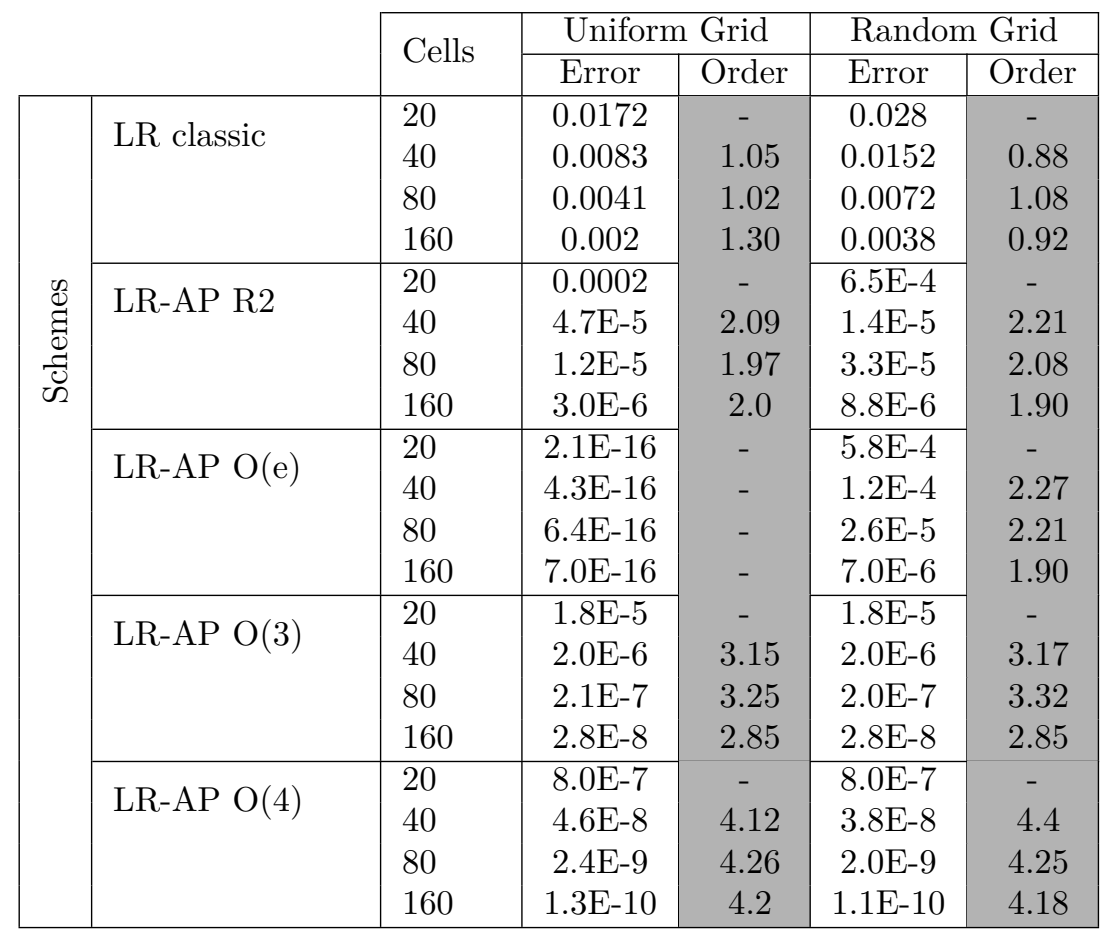

Table 3: Test case with exponential density. Err corresponds to the $L 1$ error and $q$ to the order of convergence.

Furthermore, we will extend the semi-implicit formulation and the high order reconstruction of 


\begin{tabular}{|c|c|c|c|c|c|c|}
\hline & & Colle & Uniforr & Grid & Randor & Grid \\
\hline & & Cells & Error & Order & Error & Order \\
\hline & IP ologai & 20 & 0.9001 & - & 0.8335 & - \\
\hline & Ln classic & 40 & 0.4378 & 1.05 & 0.4010 & 1.05 \\
\hline & & 80 & 0.2153 & 1.02 & 0.2065 & 0.96 \\
\hline & & 160 & 0.1067 & 1.01 & 0.1014 & 1.02 \\
\hline & & 20 & 0.1880 & - & 0.1747 & - \\
\hline 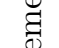 & LK-AP К & 40 & 0.0897 & 1.07 & 0.0867 & 1.01 \\
\hline ليّن & & 80 & 0.0434 & 1.05 & 0.0435 & 0.99 \\
\hline & & 160 & 0.0218 & 0.99 & 0.0218 & 0.99 \\
\hline & LR-AP R2 & 20 & 0.00894 & - & 0.0101 & - \\
\hline & & 40 & 0.0021 & 2.09 & 0.0027 & 1.91 \\
\hline & & 80 & 0.0005 & 2.07 & 7.0E-4 & 1.95 \\
\hline & & 160 & $1.2 \mathrm{E}-4$ & 2.06 & $1.7 \mathrm{E}-4$ & 2.04 \\
\hline & LB-AP O(e) & 20 & 0.0120 & - & 0.0126 & - \\
\hline & LN-AT $(\mathrm{e})$ & 40 & 0.0028 & 2.1 & 0.00332 & 1.92 \\
\hline & & 80 & 0.0007 & 2.0 & 0.00088 & 1.91 \\
\hline & & 160 & 0.00017 & 2.04 & 0.00022 & 2.04 \\
\hline & LR-AP O(3) & 20 & 0.0110 & - & 0.0079 & - \\
\hline & LR-AT U(ग) & 40 & 0.00121 & 3.18 & $8.4 \mathrm{E}-4$ & 3.23 \\
\hline & & 80 & 0.00011 & 3.46 & $7.7 \mathrm{E}-5$ & 3.45 \\
\hline & & 160 & $9.4 \mathrm{E}-6$ & 3.55 & $7.0 \mathrm{E}-6$ & 2.46 \\
\hline & LB-AP O(4) & 20 & 0.0089 & - & 0.0067 & - \\
\hline & ) & 40 & $2.1 \mathrm{E}-4$ & 5.4 & $1.5 \mathrm{E}-4$ & 5.48 \\
\hline & & 80 & $5.1 \mathrm{E}-6$ & 5.3 & $4.1 \mathrm{E}-6$ & 5.19 \\
\hline & & 160 & $2.0 \mathrm{E}-7$ & 4.67 & $1.0 \mathrm{E}-7$ & 5.36 \\
\hline
\end{tabular}

Table 4: Test case with variable gravity potential. Err corresponds to the $L 1$ error and $q$ to the order of convergence.

the equilibrium in $2 \mathrm{D}$.

\subsection{Classical Lagrange+remap scheme and notations}

The Lagrangian step is discretized with the nodal GLACE scheme defined in [10]. For the remap step we use a nodal advection scheme designed for the ALE method. We begin by introducing the notations needed.

Let us consider an unstructured mesh in dimension 2. The mesh is defined by a finite number of vertices $\mathbf{x}_{r}$ and cells $\Omega_{j}$. We denote $\mathbf{x}_{j}$ a point arbitrarily chosen inside $\Omega_{j}$. For convenience, we will call this point the center of the cell. By convention the vertices are listed counter-clockwise $\mathbf{x}_{r-1}, \mathbf{x}_{r}, \mathbf{x}_{r+1}$ with coordinates $\mathbf{x}_{r}=\left(x_{r}, y_{r}\right)$. We also define the geometric quantity

$$
\mathbf{C}_{j r}=\nabla_{\mathbf{x}_{r}}\left|\Omega_{j}\right|
$$

In dimension two, $\mathbf{C}_{j r}=l_{j r} \mathbf{n}_{j r}$ with

$$
l_{j r}=\frac{1}{2}\left\|\mathbf{x}_{r+1}-\mathbf{x}_{r-1}\right\| \text { and } \mathbf{n}_{j r}=\frac{1}{2 l_{j r}}\left(\begin{array}{c}
-y_{r-1}+y_{r+1} \\
x_{r-1}-x_{r+1}
\end{array}\right) .
$$

The convention is that the length of a vector $\mathbf{x} \in \mathbb{R}^{2}$ is denoted by $\|\mathbf{x}\|$. The scalar product of two vectors is $(\mathbf{x}, \mathbf{y})$. by

The $\mathbf{C}_{j r}$ term satisfies $\sum_{j} \mathbf{C}_{j r}=\sum_{r} \mathbf{C}_{j r}=\mathbf{0}$. The geometrical consistency of scheme is given

$$
\left|\Omega_{j}\right|=\frac{1}{d}\left(\nabla_{\mathbf{x}}\left|\Omega_{j}\right|, \mathbf{x}\right)=\frac{1}{d} \sum_{r}\left(\mathbf{C}_{j r}, \mathbf{x}_{r}\right)
$$




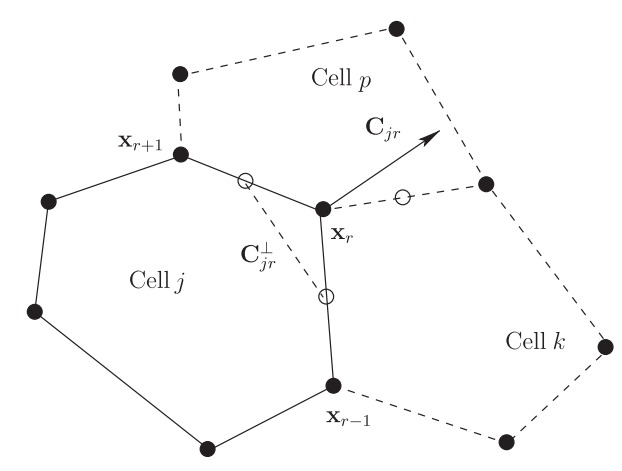

Figure 7: Notation for node formulation. The corner length $l_{j r}$ and the corner normal $\mathbf{n}_{j r}$ are defined in equation (18). Notice that $l_{j r} \mathbf{n}_{j r}$ is equal to the orthogonal vector to the half of the vector that starts at $\mathbf{x}_{r-1}$ and finish at $\mathbf{x}_{r+1}$. The center of the cell is an arbitrary point inside the cell.

To finish we define the quantities defined at the center of the cells: $\rho_{j}, \mathbf{u}_{j}, e_{j}, \tau_{j}$ and the fluxes at the nodes $\mathbf{u}_{r}, \mathbf{G}_{j r}$. The quantities at the node correspond to the average value in the cell, defined for a quantity $f$ by

$$
f_{j}=\frac{1}{\left|\Omega_{j}\right|} \int_{\left|\Omega_{j}\right|} f(t, \mathbf{x}) d \mathbf{x}
$$

For first and second order discretizations we can associate the average value in the cell to the punctual value at the middle of the cell since $\left|f\left(\mathbf{x}_{j}\right)-\frac{1}{\left|\Omega_{j}\right|} \int_{\Omega_{j}} f(t, \mathbf{x}) d \mathbf{x}\right| \leq C h^{2}$. Now we define the GLACE [10]-11] scheme for the classical Euler equation in Lagrangian formulation given by

$$
\left\{\begin{array}{l}
D_{t} \tau-\operatorname{div} \mathbf{u}=0 \\
D_{t} \mathbf{u}+\nabla p=\mathbf{0} \\
D_{t} e+\operatorname{div}(\mathbf{u} p)=0
\end{array}\right.
$$

with the material derivative $D_{t}=\partial_{t}+\mathbf{u} \cdot \nabla$. To discretize this system, we recall the GLACE scheme :

$$
\left\{\begin{array}{l}
\left|\Omega_{j}\right| \rho_{j} D_{t} \tau_{j}-\sum_{r}\left(\mathbf{C}_{j r}, \mathbf{u}_{r}\right)=0 \\
\left|\Omega_{j}\right| \rho_{j} D_{t} \mathbf{u}_{j}+\sum_{r}^{r} \mathbf{G}_{j r}=\mathbf{0} \\
\left|\Omega_{j}\right| \rho_{j} D_{t} e_{j}+\sum_{r}^{r}\left(\mathbf{G}_{j r}, \mathbf{u}_{r}\right)=0
\end{array}\right.
$$

with the fluxes

$$
\left\{\begin{array}{l}
\mathbf{G}_{j r}=p_{j} \mathbf{C}_{j r}+r_{j r} \widehat{\alpha}_{j r}\left(\mathbf{u}_{j}-\mathbf{u}_{r}\right) \\
\left(\sum_{j} r_{j r} \widehat{\alpha}_{j r}\right) \mathbf{u}_{r}=\sum_{j} p_{j} \mathbf{C}_{j r}+\sum_{j} r_{j r} \widehat{\alpha}_{j r} \mathbf{u}_{j}
\end{array}\right.
$$

where $\widehat{\alpha}_{j r}=\frac{\mathbf{C}_{j r} \otimes \mathbf{C}_{j r}}{\left|\mathbf{C}_{j r}\right|}$, and $r_{j r}$ the speed wave and $D_{t}$ the material derivative.

The first equation of (26) correspond to the 1D Riemann invariant associated with the linearized Lagrangian system, written in the direction of $\mathbf{C}_{j r}$ and multiplied by $\mathbf{C}_{j r}$. The second equation of $(26)$ is obtain by plugging the Riemann invariant in the conservative constraints $\sum_{j} \mathbf{G}_{j r}=\mathbf{0}$. Additionally, the speed wave $r_{j r}$ must be homogeneous to $\rho c$ for the Euler equation. We propose two possibilities

$$
r_{j r}=\rho_{j} c_{j}, r_{j r}=\frac{1}{N(r)} \sum_{i} \rho_{i} c_{i} .
$$


with $N(r)$ the number of cell close to the node $r$. To finish the definition of the Lagrange+remap scheme we must define an advection scheme. We propose an advection discretization using the nodal localization for the fluxes. We denote $f$ a quantity advected by the equation

$$
\partial_{t} f+\operatorname{div}(\mathbf{a} f)=0
$$

To discretize this equation we propose the following scheme

$$
\partial_{t} f_{j}+\sum_{R+}\left(\mathbf{C}_{j r}, \mathbf{a}_{r}\right) f_{j}+\sum_{R-}\left(\mathbf{C}_{j r}, \mathbf{a}_{r}\right) f_{k(r)}
$$

with $R+=\left(r /\left(\mathbf{C}_{j r}, \mathbf{a}_{r}\right)>0\right), R-=\left(r /\left(\mathbf{C}_{j r}, \mathbf{a}_{r}\right)<0\right)$ and

$$
f_{k(r)}=\frac{\sum_{j /\left(\mathbf{C}_{j r}, \mathbf{a}_{r}\right)>0}\left(\mathbf{C}_{j r}, \mathbf{a}_{r}\right) f_{j}}{\sum_{j /\left(\mathbf{C}_{j r}, \mathbf{a}_{r}\right)>0}\left(\mathbf{C}_{j r}, \mathbf{a}_{r}\right)}
$$

This scheme is conservative. For constant a the scheme is stable in norm $L^{p}$ and preserves the maximum principle. Some results are shown in the annexes. To finish we define a discrete gradient operator localized at the corner introduced and studied in [4]:

$$
\nabla_{r} f=-\left(\sum_{j} \mathbf{C}_{j r} \otimes\left(\mathbf{x}_{r}-\mathbf{x}_{j}\right)\right) \sum_{j} f_{j} \mathbf{C}_{j r}
$$

In [4, we have demonstrated that this discrete gradient is an consistant approximation of $\nabla f$ at the corner. Now we define the classical one-step Lagrange+remap scheme.

$$
\left\{\begin{array}{l}
\left|\Omega_{j}\right| \partial_{t} \rho_{j}+\left(\sum_{R_{+}} \mathbf{u}_{j r} \rho_{j}+\sum_{R_{-}} \mathbf{u}_{j r} \rho_{k(r)}\right)=0 \\
\left|\Omega_{j}\right| \partial_{t} \rho_{j} \mathbf{u}_{j}+\left(\sum_{R_{+}} \mathbf{u}_{j r}(\rho \mathbf{u})_{j}+\sum_{R_{-}} \mathbf{u}_{j r}(\rho \mathbf{u})_{k(r)}+\sum_{r} \mathbf{G}_{j r}\right)=0 \\
\left|\Omega_{j}\right| \partial_{t} \rho_{j} e_{j}+\left(\sum_{R_{+}} \mathbf{u}_{j r}(\rho e)_{j}+\sum_{R_{-}} \mathbf{u}_{j r}(\rho e)_{k(r)}+\sum_{r}\left(\mathbf{G}_{j r}, \mathbf{u}_{r}\right)\right)=0
\end{array}\right.
$$

with the fluxes defined by the problem

$$
\left\{\begin{array}{l}
\mathbf{G}_{j r}=p_{j} \mathbf{C}_{j r}+r_{j r} \widehat{\alpha}_{j r}\left(\mathbf{u}_{j}-\mathbf{u}_{r}\right) \\
\sum_{j} r_{j r} \widehat{\alpha}_{j r} \mathbf{u}_{r}=\sum_{j} p_{j} \mathbf{C}_{j r}+\sum_{j} r_{j r} \widehat{\alpha}_{j r} \mathbf{u}_{j}
\end{array}\right.
$$

The wave speed is defined by $r_{j r}=\rho_{j} c_{j}$. The expression of the flux $\mathbf{u}_{r}$ comes from a classical relation of the GLACE scheme: for the advection fluxes we define $\mathbf{u}_{j r}=\left(\mathbf{C}_{j r}, \mathbf{u}_{r}\right)$, $R_{+}=\left(r / \mathbf{u}_{j r}>0\right), R_{-}=\left(r / \mathbf{u}_{j r}<0\right)$ and $\rho_{k(r)}=\frac{\sum_{j / \mathbf{u}_{j r}>0} \mathbf{u}_{j r} \rho_{j}}{\sum_{j / \mathbf{u}_{j r}>0} \mathbf{u}_{j r}}$.

\subsection{Modified Lagrange+remap scheme for Euler equation}

In this section we propose to modify the Lagrange+remap scheme introducing the balance law between external forces and pressure effect on the fluxes. The method used is exactly the same that in 1D (Jin-Levermore procedure [19]). The balance equation between source term and hyperbolic part is $\operatorname{div}(\rho \mathbf{u} \otimes \mathbf{u})+\nabla p=-\rho \nabla \phi-\frac{\sigma}{\varepsilon^{\beta}} \rho \mathbf{u}$. But the proof of the asymptotic limit shows that $\operatorname{div}(\rho \mathbf{u} \otimes \mathbf{u})$ is homogeneous to $O\left(\varepsilon^{\gamma}\right)$ in the limit. Indeed the previous equation shows that $\mathbf{u}=$ $O\left(\varepsilon^{\beta}\right)$, consequently the important relation for the diffusion regime is $\nabla p+O\left(\varepsilon^{\gamma}\right)=-\rho \nabla \phi-\frac{\sigma}{\varepsilon^{\beta}} \rho \mathbf{u}$. 
To incorporate this relation into the fluxes we use a first order Taylor expansion and the balanced law:

$$
\begin{aligned}
& p\left(\mathbf{x}_{j}\right)=p\left(\mathbf{x}_{r}\right)+\left(\mathbf{x}_{j}-\mathbf{x}_{r}, \nabla p\left(\mathbf{x}_{r}\right)\right) \\
& p\left(\mathbf{x}_{j}\right)=p\left(\mathbf{x}_{r}\right)-\left(\mathbf{x}_{j}-\mathbf{x}_{r}, \rho\left(\mathbf{x}_{r}\right) \nabla \phi\left(\mathbf{x}_{r}\right)+\frac{\sigma}{\varepsilon \beta} \rho\left(\mathbf{x}_{r}\right) \mathbf{u}\left(\mathbf{x}_{r}\right)\right) .
\end{aligned}
$$

Now we use the the discrete equivalent of the previous equation:

$$
p_{j}=p_{j r}-\left(\mathbf{x}_{j}-\mathbf{x}_{r},(\rho \nabla \phi)_{r}+\frac{\sigma}{\varepsilon^{\beta}} \rho_{r} \mathbf{u}_{r}\right),
$$

with $(\rho \nabla \phi)_{r}$ a discretization of $\rho \nabla \phi$ at the node. Typically the first choice for $(\rho \nabla \phi)_{r}$ is $(\rho \nabla \phi)_{r}=$ $\rho_{r} \nabla_{r} \phi$ with $\rho_{r}$ average of the density around the node and $\nabla_{r} \phi$ the discrete gradient of $\phi$ at the node $r$. If we consider that $\mathbf{G}_{j r}$ is homogeneous to $p_{j r} \mathbf{C}_{j r}$, this leads to $\mathbf{G}_{j r} \approx p_{j} \mathbf{C}_{j r}-\widehat{\beta}_{j r} \rho_{r}\left(\nabla_{r} \phi+\right.$ $\left.\frac{\sigma}{\varepsilon^{\beta}} \mathbf{u}_{r}\right)$ with $\widehat{\beta}_{j r}=\mathbf{C}_{j r} \otimes\left(\mathbf{x}_{r}-\mathbf{x}_{j}\right)$. Then we get the new fluxes. We plug the previous relation in the fluxes (26). To finish we use discretization localized to the interfaces of the cells for the source term. To justify this discretization we use the following identity $\sum_{r} \widehat{\beta}_{j r}=\left|\Omega_{j}\right| \widehat{I}_{d}$ introduced in [4]. The following scheme is obtained

$$
\left\{\begin{array}{l}
\left|\Omega_{j}\right| \partial_{t} \rho_{j}+\frac{1}{\varepsilon^{\alpha}}\left(\sum_{R_{+}} \mathbf{u}_{j r} \rho_{j}+\sum_{R_{-}} \mathbf{u}_{j r} \rho_{k(r)}\right)=0 \\
\left|\Omega_{j}\right| \partial_{t} \rho_{j} \mathbf{u}_{j}+\frac{1}{\varepsilon^{\alpha}}\left(\sum_{R_{+}} \mathbf{u}_{j r}(\rho \mathbf{u})_{j}+\sum_{R_{-}} \mathbf{u}_{j r}(\rho \mathbf{u})_{k(r)}+\sum_{r} \mathbf{G}_{j r}\right) \\
=-\frac{1}{\varepsilon^{\alpha}} \sum_{r} \widehat{\beta}_{j r}\left((\rho \nabla \phi)_{r}+\frac{\sigma}{\varepsilon^{\beta}} \rho_{r} \mathbf{u}_{r}\right) \\
\left|\Omega_{j}\right| \partial_{t} \rho_{j} e_{j}+\frac{1}{\varepsilon^{\alpha}}\left(\sum_{R_{+}} \mathbf{u}_{j r}(\rho e)_{j}+\sum_{R_{-}} \mathbf{u}_{j r}(\rho e)_{k(r)}+\sum_{r}\left(\mathbf{G}_{j r}, \mathbf{u}_{r}\right)\right) \\
=-\frac{1}{\varepsilon^{\alpha}}\left(\sum_{r}\left(\widehat{\beta}_{j r}(\rho \nabla \phi)_{r}, \mathbf{u}_{r}\right)+\frac{\sigma}{\varepsilon^{\beta}} \sum_{r} \rho_{r}\left(\mathbf{u}_{r}, \widehat{\beta}_{j r} \mathbf{u}_{r}\right)\right)
\end{array}\right.
$$

with the fluxes

$$
\left\{\begin{array}{l}
\mathbf{G}_{j r}=p_{j} \mathbf{C}_{j r}+r_{j r} \widehat{\alpha}_{j r}\left(\mathbf{u}_{j}-\mathbf{u}_{r}\right)-\widehat{\beta}_{j r}\left((\rho \nabla \phi)_{r}+\frac{\sigma}{\varepsilon^{\beta}} \rho_{r} \mathbf{u}_{r}\right) \\
\left(\sum_{j} r_{j r} \widehat{\alpha}_{j r}+\frac{\sigma}{\varepsilon^{\beta}} \rho_{r} \sum_{j} \widehat{\beta}_{j r}\right) \mathbf{u}_{r}=\sum_{j} p_{j} \mathbf{C}_{j r}+\sum_{j} r_{j r} \widehat{\alpha}_{j r} \mathbf{u}_{j}-\left(\sum_{j} \widehat{\beta}_{j r}\right)(\rho \nabla \phi)_{r}
\end{array}\right.
$$

If we use an analogy with the 1D case, we have introduced the modification to obtain the $\mathrm{AP}$ property in space. As in $1 \mathrm{D}$, we have a scheme where the discretization of the source terms is non local and the friction source term associated with the momentum equation is stiff. Consequently, following the $1 \mathrm{D}$ procedure, we propose to reformulate the scheme to obtain a local implicit discretization of the friction term for the momentum equation. Firstly we introduce the $2 \mathrm{D}$ extension of the coefficient $M$ present in the $1 \mathrm{D}$ scheme. In $2 \mathrm{D}$ this coefficient comes from a local matrix associated with a node. This matrix contains the informations to modify the diffusion coefficient and the geometrical discretization of the gradient associated with the numerical viscosity. The correction by the matrix $M_{r}$ of the numerical viscosity allows us to obtain a consistant limit diffusion scheme. We define

$$
M_{r}=\left(\sum_{j} r_{j r} \widehat{\alpha}_{j r}+\frac{\sigma_{r} \rho_{r}}{\varepsilon^{\beta}} \sum_{j} \widehat{\beta}_{j r}\right)^{-1}\left(\sum_{j} r_{j r} \widehat{\alpha}_{j r}\right) .
$$


Now we notice that $\mathbf{u}_{r}=M_{r} \mathbf{u}_{r}^{*}$ and $\mathbf{u}_{r}=M_{r} \mathbf{u}_{r}^{* *}-M_{r}\left(\sum_{j} c_{j r} \widehat{\alpha}_{j r}\right)^{-1}\left(\sum_{j} \widehat{\beta}_{j r}\right)(\rho \nabla \phi)_{r}$ with $\mathbf{u}_{r}^{*}$ solution of

$$
\left(\sum_{j} r_{j r} \widehat{\alpha}_{j r}\right) \mathbf{u}_{r}^{*}=\sum_{j} p_{j} \mathbf{C}_{j r}+\sum_{j} r_{j r} \widehat{\alpha}_{j r} \mathbf{u}_{j}-\left(\sum_{j} \widehat{\beta}_{j r}\right)(\rho \nabla \phi)_{r}
$$

and $\mathbf{u}_{r}^{* *}$ solution of

$$
\left(\sum_{j} r_{j r} \widehat{\alpha}_{j r}\right) \mathbf{u}_{r}^{* *}=\sum_{j} p_{j} \mathbf{C}_{j r}+\sum_{j} r_{j r} \widehat{\alpha}_{j r} \mathbf{u}_{j}
$$

Now we introduce the definition of $\mathbf{u}_{r}$ in function of $\mathbf{u}_{r}^{* *}$ in the second equation of $(28)$. We get

$$
\mathbf{G}_{j r}=p_{j} \mathbf{C}_{j r}+r_{j r} \widehat{\alpha}_{j r}\left(\mathbf{u}_{j}-M_{r} \mathbf{u}_{r}^{* *}\right)+M_{r}\left(\sum_{j} r_{j r} \widehat{\alpha}_{j r}\right)^{-1}\left(\sum_{j} \widehat{\beta}_{j r}\right)(\rho \nabla \phi)_{r}
$$

which is equal to

$$
\mathbf{G}_{j r}=p_{j} \mathbf{C}_{j r}+r_{j r} \widehat{\alpha}_{j r} M_{r}\left(\mathbf{u}_{j}-\mathbf{u}_{r}^{* *}\right)+c_{j r}\left(\widehat{I}_{d}-M_{r}\right) \mathbf{u}_{j}+r_{j r} \widehat{\alpha}_{j r} M_{r}\left(\sum_{j} r_{j r} \widehat{\alpha}_{j r}\right)^{-1}\left(\sum_{j} \widehat{\beta}_{j r}\right)(\rho \nabla \phi)_{r}
$$

Now we study the Lagrangian fluxes of the energy equation

$$
\begin{aligned}
& \left(\mathbf{G}_{j r}, \mathbf{u}_{r}\right)=\left(p_{j} \mathbf{C}_{j r}, \mathbf{u}_{r}\right)+r_{j r}\left(\widehat{\alpha}_{j r} \mathbf{u}_{j}-M_{r} \mathbf{u}_{r}^{* *}, M_{r} \mathbf{u}_{r}^{*}\right) \\
& +\left(c_{j r} \widehat{\alpha}_{j r} M_{r}\left(\sum_{j} r_{j r} \widehat{\alpha}_{j r}\right)^{-1}\left(\sum_{j} \widehat{\beta}_{j r}\right)(\rho \nabla \phi)_{r}, M_{r} \mathbf{u}_{r}^{*}\right),
\end{aligned}
$$

with $\mathbf{u}_{r}^{*}$ the solution of the classical nodal solver. The previous equation is equivalent to

$$
\begin{gathered}
\left(\mathbf{G}_{j r}, \mathbf{u}_{r}\right)=\left(p_{j} \mathbf{C}_{j r}, \mathbf{u}_{r}\right)+r_{j r}\left(\widehat{\alpha}_{j r} M_{r}\left(\mathbf{u}_{j}-\mathbf{u}_{r}^{* *}\right), M_{r} \mathbf{u}_{r}^{*}\right)+r_{j r} \widehat{\alpha}_{j r}\left(\left(\widehat{I}_{d}-M_{r}\right) \mathbf{u}_{j}, M_{r} \mathbf{u}_{r}^{*}\right) \\
+r_{j r}\left(\widehat{\alpha}_{j r} M_{r}\left(\sum_{j} r_{j r} \widehat{\alpha}_{j r}\right)^{-1}\left(\sum_{j} \widehat{\beta}_{j r}\right)(\rho \nabla \phi)_{r}, M_{r} \mathbf{u}_{r}^{*}\right) .
\end{gathered}
$$

Now we plug this manipulation in the scheme to obtain an other formulation with a source term with a variable localized at the center of cell. To finish we use a semi-implicit time discretization

Definition 3.1 (Semi discrete LR-AP scheme). The semi-discrete Lagrange+remap AP scheme is given by

$$
\left\{\begin{array}{l}
\left|\Omega_{j}\right| \partial_{t} \rho_{j}+\frac{1}{\varepsilon^{\alpha}}\left(\sum_{R+}\left(\mathbf{C}_{j r}, M_{r} \mathbf{u}_{r}^{*}\right) \rho_{j}+\sum_{R-}\left(\mathbf{C}_{j r}, M_{r} \mathbf{u}_{r}^{*}\right) \rho_{k(r)}\right)=0 \\
\left|\Omega_{j}\right| \partial_{t} \rho_{j} \mathbf{u}_{j}+\frac{1}{\varepsilon^{\alpha}}\left(\sum_{R+}\left(\mathbf{C}_{j r}, M_{r} \mathbf{u}_{r}^{*}\right)(\rho \mathbf{u})_{j}+\sum_{R-}\left(\mathbf{C}_{j r}, M_{r} \mathbf{u}_{r}^{*}\right)(\rho \mathbf{u})_{k(r)}+\sum_{r} \mathbf{G}_{j r}\right)= \\
-\frac{1}{\varepsilon^{\alpha}}\left(\sum_{r} r_{j r} \widehat{\alpha}_{j r} N_{r} M_{r}(\rho \nabla \phi)_{r}\right)-\frac{1}{\varepsilon^{\alpha}}\left(\sum_{r} r_{j r} \widehat{\alpha}_{j r}\left(\widehat{I}_{d}-M_{r}\right)\right) \mathbf{u}_{j} \\
\left|\Omega_{j}\right| \partial_{t} \rho_{j} e_{j}+\frac{1}{\varepsilon^{\alpha}}\left(\sum_{R+}\left(\mathbf{C}_{j r}, M_{r} \mathbf{u}_{r}^{*}\right)(\rho e)_{j}+\sum_{R-}\left(\mathbf{C}_{j r}, M_{r} \mathbf{u}_{r}^{*}\right)(\rho e)_{k(r)}+\sum_{r}\left(\mathbf{G}_{j r}, M_{r} \mathbf{u}_{r}^{*}\right)\right) \\
=-\frac{1}{\varepsilon^{\alpha}}\left(\sum_{r} r_{j r}\left(\widehat{\alpha}_{j r} M_{r} N_{r}(\rho \nabla \phi)_{r}, M_{r} \mathbf{u}_{r}^{*}\right)\right)-\frac{1}{\varepsilon^{\alpha}}\left(\sum_{r} r_{j r} \widehat{\alpha}_{j r}\left(\left(\widehat{I_{d}}-M_{r}\right) \mathbf{u}_{j}, M_{r} \mathbf{u}_{r}^{*}\right)\right)
\end{array}\right.
$$


with the fluxes

$$
\left\{\begin{array}{l}
\mathbf{G}_{j r}=p_{j} \mathbf{C}_{j r}+r_{j r} \widehat{\alpha}_{j r} M_{r}\left(\mathbf{u}_{j}-\mathbf{u}_{r}^{* *}\right) \\
\left(\sum_{j} r_{j r} \widehat{\alpha}_{j r}\right) \mathbf{u}_{r}^{*}=\sum_{j} p_{j} \mathbf{C}_{j r}+\sum_{j} r_{j r} \widehat{\alpha}_{j r} \mathbf{u}_{j}-\left(\sum_{j} \widehat{\beta}_{j r}\right)(\rho \nabla \phi)_{r} \\
\left.\sum_{j} r_{j r} \widehat{\alpha}_{j r}\right) \mathbf{u}_{r}^{* *}=\sum_{j} p_{j} \mathbf{C}_{j r}+\sum_{j} r_{j r} \widehat{\alpha}_{j r} \mathbf{u}_{j}
\end{array}\right.
$$
$\rho_{j}$.

with $\widehat{\alpha}_{j r}=\frac{\mathbf{C}_{j r} \otimes \mathbf{C}_{j r}}{\left|\mathbf{C}_{j r}\right|}, \widehat{\beta}_{j r}=\mathbf{C}_{j r} \otimes\left(\mathbf{x}_{r}-\mathbf{x}_{j}\right), N_{r}=\left(\sum_{j} r_{j r} \widehat{\alpha}_{j r}\right)^{-1}\left(\sum_{j} \widehat{\beta}_{j r}\right)$ and $\rho_{r}$ a mean of

Now we introduce the fully-discrete scheme where we use a implicit discretization for the friction source term.

Definition 3.2 (Fully discrete LR-AP scheme). The final Lagrange+remap AP scheme (semi implicit in time) is given by

$$
\left\{\begin{array}{l}
\left|\Omega_{j}\right| \frac{\rho_{j}^{n+1}-\rho_{j}^{n}}{\Delta t}+\frac{1}{\varepsilon^{\alpha}}\left(\sum_{R+}\left(\mathbf{C}_{j r}, M_{r} \mathbf{u}_{r}^{*}\right) \rho_{j}^{n}+\sum_{R-}\left(\mathbf{C}_{j r}, M_{r} \mathbf{u}_{r}^{*}\right) \rho_{k(r)}^{n}\right)=0 \\
\left|\Omega_{j}\right| \frac{\left(\rho_{j} \mathbf{u}_{j}\right)^{n+1}-\left(\rho_{j} \mathbf{u}_{j}\right)^{n}}{\Delta t}+\frac{1}{\varepsilon^{\alpha}}\left(\sum_{R+}\left(\mathbf{C}_{j r}, M_{r} \mathbf{u}_{r}^{*}\right)(\rho \mathbf{u})_{j}^{n}+\sum_{R-}\left(\mathbf{C}_{j r}, M_{r} \mathbf{u}_{r}^{*}\right)(\rho \mathbf{u})_{k(r)}^{n}+\sum_{r} \mathbf{G}_{j r}\right)= \\
-\frac{1}{\varepsilon^{\alpha}}\left(\sum_{r} r_{j r} \widehat{\alpha}_{j r} M_{r} N_{r}(\rho \nabla \phi)_{r}\right)-\frac{1}{\varepsilon^{\alpha}}\left(\sum_{r} r_{j r} \widehat{\alpha}_{j r}\left(\widehat{I}_{d}-M_{r}\right)\right) \mathbf{u}_{j}^{n+1} \\
\left|\Omega_{j}\right| \frac{\rho_{j} e_{j}^{n+1}-\left(\rho_{j} e_{j}\right)^{n}}{\Delta t}+\frac{1}{\varepsilon^{\alpha}}\left(\sum_{R+}\left(\mathbf{C}_{j r}, M_{r} \mathbf{u}_{r}^{*}\right)(\rho e)_{j}^{n}+\sum_{R-}\left(\mathbf{C}_{j r}, M_{r} \mathbf{u}_{r}^{*}\right)(\rho e)_{k(r)}^{n}+\sum_{r}\left(\mathbf{G}_{j r}, M_{r} \mathbf{u}_{r}^{*}\right)\right) \\
=-\frac{1}{\varepsilon^{\alpha}}\left(\sum_{r} r_{j r}\left(\widehat{\alpha}_{j r} M_{r} N_{r}(\rho \nabla \phi)_{r}, M_{r} \mathbf{u}_{r}^{*}\right)\right)-\frac{1}{\varepsilon^{\alpha}}\left(\sum_{r} r_{j r} \widehat{\alpha}_{j r}\left(\left(\widehat{I}_{d}-M_{r}\right) \mathbf{u}_{j}^{n}, M_{r} \mathbf{u}_{r}^{*}\right)\right)
\end{array}\right.
$$

with the fluxes

$$
\left\{\begin{array}{l}
\mathbf{G}_{j r}=p_{j}^{n} \mathbf{C}_{j r}+r_{j r} \widehat{\alpha}_{j r} M_{r}\left(\mathbf{u}_{j}^{n}-\mathbf{u}_{r}^{* *}\right) \\
\left(\sum_{j} r_{j r} \widehat{\alpha}_{j r}\right) \mathbf{u}_{r}^{*}=\sum_{j} p_{j}^{n} \mathbf{C}_{j r}+\sum_{j} r_{j r} \widehat{\alpha}_{j r} \mathbf{u}_{j}-\left(\sum_{j} \widehat{\beta}_{j r}\right)(\rho \nabla \phi)_{r} \\
\left.\sum_{j} r_{j r} \widehat{\alpha}_{j r}\right) \mathbf{u}_{r}^{* *}=\sum_{j} p_{j}^{n} \mathbf{C}_{j r}+\sum_{j} r_{j r} \widehat{\alpha}_{j r} \mathbf{u}_{j}^{n}
\end{array}\right.
$$
$\rho_{j}$.

with $\widehat{\alpha}_{j r}=\frac{\mathbf{C}_{j r} \otimes \mathbf{C}_{j r}}{\left|\mathbf{C}_{j r}\right|}, \widehat{\beta}_{j r}=\mathbf{C}_{j r} \otimes\left(\mathbf{x}_{r}-\mathbf{x}_{j}\right), N_{r}=\left(\sum_{j} r_{j r} \widehat{\alpha}_{j r}\right)^{-1}\left(\sum_{j} \widehat{\beta}_{j r}\right)$ and $\rho_{r}$ a mean of

This scheme is also asymptotic preserving. As the linear case, it admits a CFL condition independent of $\varepsilon$ for $\beta=1$ (this point is verified only numerically). A study on Cartesian meshes show that $1-M_{r}=O\left(\frac{\sigma}{\varepsilon^{\beta}} M_{r}\right)$. Since $M_{r}=O\left(\varepsilon^{\beta}\right)$, we remark that the fluxes and the source term for the last equation are homogeneous to $O(1)$. For the equation on the momentum, the fluxes are homogeneous to $O(1)$, and the source term is homogeneous to $O\left(\frac{1}{\varepsilon^{\beta}}\right)$. Using an implicit discretization for the source term, we have a scheme with a CFL condition independent of $\varepsilon$.

Now we propose to proof the asymptotic limit for the explicit scheme (30)-31). We use the complete equivalent form of the scheme (27)-287. 
Proposition 3.1. We assume that the local matrix is invertible and the density is positive. We assume that $\alpha=\beta=1$. The scheme $L R-A P$ 27)-28) for the euler equations tends to the following diffusion scheme when $\varepsilon$ tend to zero

$$
\left\{\begin{array}{l}
\left|\Omega_{j}\right| \partial_{t} \rho_{j}+\left(\sum_{R+} \mathbf{u}_{j r} \rho_{j}+\sum_{R-} \mathbf{u}_{j r} \rho_{k(r)}\right)=0 \\
\left|\Omega_{j}\right| \partial_{t} \rho_{j} e_{j}+\left(\sum_{R+} \mathbf{u}_{j r}(\rho e)_{j}+\sum_{R-} \mathbf{u}_{j r}(\rho e)_{k(r)}+p_{j} \sum_{r}\left(\mathbf{C}_{j r}, \mathbf{u}_{r}\right)\right)=0 \\
\sigma_{r} \rho_{r}\left(\sum_{j} \widehat{\beta}_{j r}\right) \mathbf{u}_{r}=\sum_{j} p_{j} \mathbf{C}_{j r}-\left(\sum_{j} \widehat{\beta}_{j r}\right)(\rho \nabla \phi)_{r}
\end{array}\right.
$$

with $\mathbf{u}_{j r}=\left(\mathbf{u}_{r}, \mathbf{C}_{j r}\right)$.

Proof. To obtain this result, we use a Hilbert expansion and the second form of the scheme 27 - 28, . We begin by simplifying the source term with the last part of the fluxes $\sum_{r} \mathbf{G}_{j r}$ and $\sum_{r}\left(\mathbf{G}_{j r}, \mathbf{u}_{r}\right)$. We get a scheme without term source. Following, we plug these definitions in the model and we observe the term homogeneous to $\frac{1}{\varepsilon^{m}}$.

The terms homogeneous to $\frac{1}{\varepsilon}$ are

$$
\sigma_{r} \rho_{r}^{0}\left(\sum_{r} \widehat{\beta}_{j r}\right) \mathbf{u}_{r}^{0}=\mathbf{0}
$$

Since the density is positive then $\mathbf{u}_{r}=\mathbf{0}$ and

$$
\begin{aligned}
& \sum_{R+} \mathbf{u}_{j r}^{0} \rho_{j}^{0}+\sum_{R-} \mathbf{u}_{j r}^{0} \rho_{k(r)}^{0}=0 \\
& \sum_{R+}^{R+} \mathbf{u}_{j r}^{0} \rho_{j}^{0} \mathbf{u}_{j}^{0}+\sum_{R-} \mathbf{u}_{j r}^{0} \rho_{k(r)}^{0} \mathbf{u}_{k(r)}+\sum_{r} p_{j}^{0} \mathbf{C}_{j r}+\sum_{r} r_{j r}^{0} \widehat{\alpha}_{j r}\left(\mathbf{u}_{j}^{0}-\mathbf{u}_{r}^{0}\right)=\mathbf{0} \\
& \sum_{R+}^{R} \mathbf{u}_{j r}^{0} \rho_{j}^{0} e_{j}^{0}+\sum_{R-} \mathbf{u}_{j r}^{0} \rho_{k(r)}^{0} e_{k(r)}+\sum_{r}\left(p_{j}^{0} \mathbf{C}_{j r}, \mathbf{u}_{r}^{0}\right)+\sum_{r} r_{j r}^{0} \widehat{\alpha}_{j r}\left(\left(\mathbf{u}_{j}^{0}-\mathbf{u}_{r}^{0}\right), \mathbf{u}_{r}^{0}\right)=0
\end{aligned}
$$

Since we have assume that the matrix is invertible and that the density is positive then $\mathbf{u}_{r}^{0}=\mathbf{0}$. Using this result and knowing $\sum_{r} \mathbf{C}_{j r}=\mathbf{0}$ (property of nodal schemes) the second equation gives

$$
\sum_{r} r_{j r}^{0} \widehat{\alpha}_{j r} \mathbf{u}_{j}^{0}=\mathbf{0}
$$

A study analogue to the result gives, in previous works for $\sum_{j} \widehat{\alpha}_{j r}$, show that this matrix is invertible. Due to $\rho_{j}>0$ we can assert that $\mathbf{u}_{j}^{0}=\mathbf{0}$. To finish we give the terms homogeneous to 1 . To simplify these terms we use the fact that $\mathbf{u}_{r}^{0}=\mathbf{0}$ and $\mathbf{u}_{j}^{0}=\mathbf{0}$.

The terms homogeneous to $\frac{1}{\varepsilon^{0}}$ are

$$
\begin{aligned}
& \partial_{t}\left|\Omega_{j}\right| \rho_{j}^{0}+\sum_{R+} \mathbf{u}_{j r}^{1} \rho_{j}^{0}+\sum_{R-} \mathbf{u}_{j r}^{1} \rho_{k(r)}^{0}=0 \\
& \sum_{r} \mathbf{C}_{j r} p_{j}^{1}+\sum_{r} \widehat{\alpha}_{j r} r_{j r}^{0}\left(\mathbf{u}_{j}^{1}-\mathbf{u}_{r}^{1}\right)=\mathbf{0} \\
& \partial_{t}\left|\Omega_{j}\right| \rho_{j}^{0} e_{j}^{0}+\sum_{r} \mathbf{C}_{j r}\left(p_{j}^{0}, \mathbf{u}_{r}^{1}\right)+\sum_{r}\left(\widehat{\alpha}_{j r} r_{j r}^{0}\left(\mathbf{u}_{j}^{0}-\mathbf{u}_{r}^{0}\right), \mathbf{u}_{r}^{1}\right)=0
\end{aligned}
$$

and, since $\mathbf{u}_{r}^{0}=\mathbf{0}, \mathbf{u}_{j}^{0}=\mathbf{0}$ and $\sum_{r} \mathbf{C}_{j r} p_{j}^{1}=0$, we can write 


$$
\left(\sigma_{r} \rho_{r}^{0} \widehat{\beta}_{j r}\right) \mathbf{u}_{r}^{1}=\sum_{j} p_{j}^{0} \mathbf{C}_{j r}-\sum_{j} \widehat{\beta}_{j r}(\rho \nabla \phi)_{r}
$$

To finish we couple the first and third equations of (37) and (36).

\subsection{Entropy property}

In this section we want study the entropy properties associated with the scheme. More precisely how the entropy inequality is modified in the new AP scheme. Actually we cannot give a full result about the entropy stability for the fully discrete scheme. Thus, we propose to give the entropy inequality on positivity condition for the scheme continuous in time. This result is not sufficient but gives an idea on the modification introduced in the entropy inequality. We introduce the Lagrangian system with friction and gravity:

$$
\left\{\begin{array}{l}
\rho D_{t} \tau-\frac{1}{\varepsilon^{\alpha}} \operatorname{div}(\mathbf{u})=0 \\
\rho D_{t} \mathbf{u}+\frac{1}{\varepsilon^{\alpha}} \nabla p=-\frac{1}{\varepsilon^{\alpha}} \nabla \phi-\frac{\sigma}{\varepsilon^{\beta}} \mathbf{u} \\
\rho D_{t} e+\frac{1}{\alpha} \operatorname{div}(p \mathbf{u})=-\frac{1}{\varepsilon^{\alpha}}(\nabla \phi, \mathbf{u})-\frac{\sigma}{\varepsilon^{\beta}}(\mathbf{u}, \mathbf{u})
\end{array}\right.
$$

with the material derivative $D_{t}=\partial_{t}+\frac{1}{\varepsilon^{\alpha}} \mathbf{u} . \nabla$. Now we introduce the physical concave entropy $S$ and the second principle of thermodynamic

$$
T D_{t} S=D_{t} e-\left(\mathbf{u}, D_{t} \mathbf{u}\right)+p D_{t} \tau,
$$

multiplying the equation by $\rho$ and using the systems we obtain

$$
T \rho D_{t} S=-\frac{1}{\varepsilon^{\alpha}}(\nabla \phi, \mathbf{u})-\frac{\sigma}{\varepsilon^{\beta}}(\mathbf{u}, \mathbf{u})+\frac{1}{\varepsilon^{\alpha}}(\nabla \phi, \mathbf{u})+\frac{\sigma}{\varepsilon^{\beta}}(\mathbf{u}, \mathbf{u})=0 .
$$

Therefore, we have the classical entropy equality for Lagrangian system. Now we have

$$
\partial_{t}(\rho S)+\frac{1}{\varepsilon^{\alpha}} \operatorname{div}(\rho \mathbf{u} S)=\rho D_{t} S+S\left(\partial_{t} \rho+\frac{1}{\varepsilon^{\alpha}} \operatorname{div}(\rho \mathbf{u})\right) .
$$

Using the Eulerian equations and $\rho D_{t} S=0$, the following entropy equality yields

$$
\partial_{t}(\rho S)+\frac{1}{\varepsilon^{\alpha}} \operatorname{div}(\rho \mathbf{u} S)=0,
$$

for the smooth solution and

$$
\partial_{t}(\rho S)+\frac{1}{\varepsilon^{\alpha}} \operatorname{div}(\rho \mathbf{u} S) \geq 0 .
$$

for the discontinuous solutions.

To finish using the different equations of the Euler equations (2), we obtain easily the following conservation law on the internal energy $\epsilon=e-\frac{1}{2}|\mathbf{u}|^{2}$ :

$$
\partial_{t}(\rho \epsilon)+\operatorname{div}(\rho \mathbf{u} \epsilon)=0 .
$$

Now we want prove that the semi discrete scheme in space (discrete in space, continuous in time) satisfies the entropy inequality under positivity condition.

Lemma 3.2. If $\rho_{j}(t)>0$ and $T_{j}(t)>0$ the scheme LR-AP (27)-(28) is entropic, hence

$$
\left|\Omega_{j}\right| \partial_{t}\left(\rho_{j} S_{j}\right)+\frac{1}{\epsilon^{\alpha}}\left(\sum_{R^{+}}\left(\mathbf{u}_{r}, \mathbf{C}_{j r}\right) \rho_{j} S_{j}+\sum_{R^{-}}\left(\mathbf{u}_{r}, \mathbf{C}_{j r}\right) \rho_{k(r)} S_{k(r)}\right) \geq 0
$$

and the density and the energy are positive for all time. 
Proof. Firsly we use the second principle of the thermodynamic to obtain an equation on $\partial_{t}\left(\rho_{j} S_{j}\right)$

$$
T \partial_{t} S=\partial_{t} e-\left(\mathbf{u}, \partial_{t} \mathbf{u}\right)+q \partial_{t} \tau
$$

which yields

$$
\partial_{t}(\rho S)=\frac{1}{T}\left(\rho \partial_{t} e-\left(\mathbf{u}, \rho \partial_{t} \mathbf{u}\right)+\rho q \partial_{t} \tau\right)+S \partial_{t} \rho
$$

and we have

$$
\partial_{t}(\rho S)=\frac{1}{T}\left(\partial_{t}(\rho e)-\left(\mathbf{u}, \partial_{t}(\rho \mathbf{u})\right)+(S T-e+(\mathbf{u}, \mathbf{u})-q \tau) \partial_{t} \rho\right) .
$$

Now, using 42 and 41, we deduce

$$
\begin{gathered}
\left|\Omega_{j}\right| \frac{1}{T_{j}}\left(\partial_{t}\left(\rho_{j} e_{j}\right)-\left(\mathbf{u}_{j}, \partial_{t}\left(\rho_{j} \mathbf{u}_{j}\right)\right)+\left(S_{j} T_{j}-e_{j}+\left(\mathbf{u}_{j}, \mathbf{u}_{j}\right)-q_{j} \tau_{j}\right) \partial_{t} \rho_{j}\right) \\
+\frac{1}{\varepsilon^{\alpha}}\left(\sum_{R^{+}} l_{j r}\left(\mathbf{u}_{r}, \mathbf{n}_{j r}\right) \rho_{j} S_{j}+\sum_{R^{-}} l_{j r}\left(\mathbf{u}_{r}, \mathbf{n}_{j r}\right)(\rho S)_{k(r)}\right)
\end{gathered}
$$

Using the scheme, we incorporate the spacial discretization in the previous equations. We split the equations obtained between the Lagrangian fluxes and the Eulerian fluxes. Consequently $43=(L+P)$ where $L$ the Lagrangian fluxes and $P$ the Eulerian fluxes.

$$
\begin{aligned}
L & =\frac{1}{T_{j} \varepsilon^{\alpha}}\left(\left(\sum_{r} \mathbf{G}_{j r}, \mathbf{u}_{j}\right)+\sum_{r} \widehat{\beta}_{j r}\left((\rho \nabla \phi)_{r}+\frac{\sigma_{r}}{\varepsilon^{\beta}} \rho_{r} \mathbf{u}_{r}, \mathbf{u}_{j}\right)-\sum_{r}\left(\mathbf{G}_{j r}, \mathbf{u}_{r}\right)\right. \\
& -\sum_{r}\left(\hat{\beta}_{j r}\left((\rho \nabla \phi)_{r}+\frac{\sigma_{r}}{\varepsilon^{\beta}} \rho_{r} \mathbf{u}_{r}, \mathbf{u}_{r}\right)+\sum_{r} p_{j}\left(\mathbf{u}_{r}, \mathbf{C}_{j r}\right)-\sum_{r} p_{j}\left(\mathbf{u}_{r}, \mathbf{C}_{j r}\right)\right)
\end{aligned}
$$

We note that

$$
\sum_{r}\left(\mathbf{G}_{j r}, \mathbf{u}_{r}\right)=\sum_{r} p_{j}\left(\mathbf{C}_{j r}, \mathbf{u}_{r}\right)+r_{j r}\left(\hat{\alpha}_{j r}\left(\mathbf{u}_{j}-\mathbf{u}_{r}\right), \mathbf{u}_{r}\right)-\hat{\beta}_{j r}\left((\rho \nabla \phi)_{r}+\frac{\sigma_{r}}{\varepsilon^{\beta}} \rho_{r} \mathbf{u}_{r}, \mathbf{u}_{r}\right)
$$

Which gives

$$
\sum_{r}\left(\mathbf{G}_{j r}, \mathbf{u}_{r}\right)+\sum_{r}\left(\hat{\beta}_{j r}\left((\rho \nabla \phi)_{r}+\frac{\sigma_{r}}{\varepsilon^{\beta}} \rho_{r} \mathbf{u}_{r}, \mathbf{u}_{r}\right)=\sum_{r} p_{j}\left(\mathbf{C}_{j r}, \mathbf{u}_{r}\right)-r_{j r}\left(\hat{\alpha}_{j r}\left(\mathbf{u}_{j}-\mathbf{u}_{r}\right), \mathbf{u}_{r}\right)\right.
$$

the other term satisfies

$$
\left(\sum_{r}\left(\mathbf{G}_{j r}, \mathbf{u}_{j}\right)+\sum_{r} \widehat{\beta}_{j r}\left((\rho \nabla \phi)_{r}+\frac{\sigma_{r}}{\varepsilon^{\beta}} \rho_{r} \mathbf{u}_{r}, \mathbf{u}_{j}\right)\right)=\sum_{r} p_{j}\left(\mathbf{C}_{j r}, \mathbf{u}_{j}\right)+r_{j r}\left(\hat{\alpha}_{j r}\left(\mathbf{u}_{j}-\mathbf{u}_{r}\right), \mathbf{u}_{j}\right)
$$

Using that $\sum_{r} \mathbf{C}_{j r}=0$, we have

$$
L=\frac{1}{T_{j} \varepsilon}\left(\sum_{r} c_{j r}\left(\mathbf{C}_{j r},\left(\mathbf{u}_{j}-\mathbf{u}_{r}\right)\right)^{2}\right)-\frac{p_{j}}{T_{j} \varepsilon} \sum_{r}\left(\mathbf{u}_{r}, \mathbf{C}_{j r}\right)
$$

Now we study the Eulerian fluxes with $-\frac{p_{j}}{T_{j} \varepsilon} \sum_{r}\left(\mathbf{u}_{r}, \mathbf{C}_{j r}\right)$. This term is close to the upwind scheme with $p_{j}$ at the place of $p_{k(r)}$. We factorize and get $P+\frac{p_{j}}{T_{j} \varepsilon} \sum_{r} \mathbf{u}_{j r}=A+B$ with

$$
\begin{aligned}
& A=\frac{1}{T_{j} \varepsilon^{\alpha}} \sum_{R^{+}} \mathbf{u}_{j r}\left(T_{j} \rho_{j} S_{j}-\rho_{j} e_{j}+\left(\mathbf{u}_{j}, \rho_{j} \mathbf{u}_{j}\right)-\left(S_{j} T_{j}-e_{j}+\left(\mathbf{u}_{j}, \mathbf{u}_{j}\right)-\tau_{j} p_{j}\right) \rho_{j}-p_{j}\right) \\
& B=\frac{1}{T_{j} \varepsilon^{\alpha}} \sum_{R^{-}} \mathbf{u}_{j r}\left(T_{j}(\rho S)_{k(r)}-(\rho e)_{k(r)}+\left(\mathbf{u}_{j},(\rho \mathbf{u})_{k(r)}\right)-\left(S_{j} T_{j}-e_{j}+\left(\mathbf{u}_{j}, \mathbf{u}_{j}\right)-\tau_{j} p_{j}\right) \rho_{k(r)}-p_{j}\right)
\end{aligned}
$$


We have two cases, $\mathbf{u}_{j r}$ is positive or negative. The term $A$ is equal to zero. Now we study the term $B$ with $\mathbf{u}_{j r}$ negative. We begin with the term

$$
\sum_{R^{-}} \mathbf{u}_{j r}\left(T_{j}(\rho S)_{k r}-(\rho e)_{k(r)}+\left(\mathbf{u}_{j},(\rho \mathbf{u})_{k(r)}\right)-\left(S_{j} T_{j}-e_{j}+\left(\mathbf{u}_{j}, \mathbf{u}_{j}\right)-\tau_{j} p_{j}\right) \rho_{k(r)}-p_{j}\right)
$$

We know that

$$
\left(T_{j} \rho_{j} S_{j}-\rho_{j} e_{j}+\left(\mathbf{u}_{j}, \rho_{j} \mathbf{u}_{j}\right)-\left(S_{j} T_{j}-e_{j}+\left(\mathbf{u}_{j}, \mathbf{u}_{j}\right)-\tau_{j} p_{j}\right) \rho_{j}-p_{j}\right)=0
$$

We subtract the previous term to 47). Hence the term inside the sum in 47) is equal to

$$
\begin{aligned}
B= & \frac{1}{T_{j} \varepsilon^{\alpha}}\left((\rho S)_{k(r)}-\rho_{j} S_{j}\right)-\frac{1}{T_{j} \varepsilon}\left((\rho e)_{k(r)}-\rho_{j} e_{j}\right)+\frac{1}{T_{j} \varepsilon}\left(\mathbf{u}_{j},(\rho \mathbf{u})_{k(r)}-\rho_{j} \mathbf{u}_{j}\right) \\
& -\frac{1}{T_{j} \varepsilon^{\alpha}}\left(S_{j} T_{j}-e_{j}+\left(\mathbf{u}_{j}, \mathbf{u}_{j}\right)-\tau_{j} p_{j}\right)\left(\rho_{k(r)}-\rho_{j}\right)
\end{aligned}
$$

We note that $H(X)=\rho_{j} S_{j}$ and $X=\left(\rho_{j}, \rho_{j} \mathbf{u}_{j}, \rho_{j} e_{j}\right)$. Furthermore, $\nabla H=\frac{1}{T_{j}}\left(S_{j} T_{j}-e_{j}+\right.$ $\left.\left(\mathbf{u}_{j}, \mathbf{u}_{j}\right)-\tau_{j} p_{j},-\mathbf{u}_{j}, 1\right)$. Since the entropy is concave

$$
H\left(\mathbf{X}_{k}\right)-H\left(\mathbf{X}_{i}\right)-\left(\mathbf{X}_{k}-\mathbf{X}_{j}, \nabla H\left(\mathbf{x}_{j}\right)\right) \leq 0
$$

This is the relation which is present in the previous expression of $B$. This term is negative but $\left(\mathbf{u}_{r}, \mathbf{C}_{j r}\right)<0$, thus 477 is positive. At the end, since $P+\frac{p_{j}}{T_{j} \varepsilon^{\alpha}} \sum_{r} \mathbf{u}_{j r}>0$ and using 466 , it yields that $L+P$ is positive. Consequently, if $\rho_{j}>0$ and $T_{j}>0$ is positive, the entropy inequality is satisfied.

The semi-discrete AP scheme (continuous in time, discrete in space) satisfies the entropy inequality. We note that the modification in space does not modify the entropy inequality, indeed the positive terms in the proof have the same form that for the classical scheme only the nodal velocity $\mathbf{u}_{r}$ chance between the two scheme. In conclusion, we deduce that with a slightly different CFL than the classical scheme's CFL, the fully discrete AP scheme [still?] satisfies the entropy inequality. Nevertheless, this still remains to be proved.

\subsection{Discretization of the steady states}

Not unlike the 1D case, we will study the discretization of the steady states (4, by the LR-AP scheme. For the steady steady state (4) the situation is the same tat in 1D the local Matrix $M_{r}$ in the scheme 32,33 allows to obtain a nodal velocity homogeneous to the physical velocity ( in order to $\varepsilon^{\beta}$ ). Now we consider the second subset of steady state (5). The aim is to obtain a scheme with a high accuracy around the hydrostatic equilibrium configuration $(\nabla p=\rho \nabla \phi$ and $\mathbf{u}=\mathbf{0})$. In this section we show that the AP scheme satisty the well-balanced property [13] and is more efficient to treat these configurations. We assume that the initial data $\left(\rho_{j}, \mathbf{u}_{j}, e_{j}\right)$ satisfies the discrete steady state at the interface $\left(\nabla_{r} p=-(\rho \nabla \phi)_{r}\right.$ for Euler equations). As in 1D, a scheme is well-balanced if the scheme is exact for the discrete steady state. For the Shallow water equations the discrete steady state is an exact discretization of the continuous steady states. This is not the case for the Euler equations. That is the reason why the well balanced scheme are not exact for the steady states of the Euler equations. However the numerical error of the well-balanced scheme for the Euler equations, comes only from the error between continuous and discrete steady state.

Lemma 3.3. Assume the initial data is given by $\mathbf{u}_{j}=\mathbf{0}$ and $\nabla_{r} p=-(\rho \nabla \phi)_{r}$ which is equivalent to

$$
\nabla_{r} p=-\left(\sum_{j} \widehat{\beta}_{j r}\right)^{-1} \sum_{j} p_{j} \mathbf{C}_{j r}=-(\rho \nabla \phi)_{r}
$$

with $\rho_{r}$ a mean of $\rho_{j}$ around $r$. Then the semi-implicit (as well as, the explicit) LR-AP scheme is stationary for the hydrostatic equilibrium. 
Proof. By definition of the initialization we know that $\sum_{j} p_{j} \mathbf{C}_{j r}=\left(\sum_{j} \widehat{\beta}_{j r}\right)(\rho \nabla \phi)_{r}$. Plugging this relation in the definition $\mathbf{u}_{r}^{*}$ we obtain

$$
\left(\sum_{j} r_{j r} \widehat{\alpha}_{j r}\right) \mathbf{u}_{r}^{*}=\left(\sum_{j} r_{j r} \widehat{\alpha}_{j r}\right) \mathbf{u}_{j} .
$$

Since $\mathbf{u}_{j}=\mathbf{0}$ then $\mathbf{u}_{r}^{*}=\mathbf{0}$. Using the same computations we have $\mathbf{u}_{r}^{* *}=\rho_{r} N_{r} \nabla_{r} \phi$. Using the values of $\mathbf{u}_{r}^{*}$ and $\mathbf{u}_{r}^{* *}$ in the scheme we get $\rho_{j}^{n+1}=\rho_{j}^{n},(\rho e)^{n+1}=(\rho e)^{n}$ and

$$
\frac{(\rho \mathbf{u})^{n+1}-(\rho \mathbf{u})^{n}}{\Delta t}=-\frac{1}{\varepsilon^{\alpha}} \sum_{r} \mathbf{G}_{j r}-\frac{1}{\varepsilon^{\alpha}}\left(\sum_{r} c_{j r} \widehat{\alpha}_{j r} M_{r} N_{r} \rho_{r} \nabla_{r} \phi\right)-\frac{1}{\varepsilon^{\alpha}}\left(\sum_{r} c_{j r} \widehat{\alpha}_{j r}\left(\widehat{I}_{d}-M_{r}\right)\right) \mathbf{u}_{j}^{n+1}
$$

By definition of $\mathbf{u}_{r}^{* *}$, it is known that $\sum_{r} \mathbf{G}_{j r}=-\sum_{r} c_{j r} \widehat{\alpha}_{j r} M_{r} N_{r} \rho_{r} \nabla_{r} \phi$ which leads to

$$
\frac{(\rho \mathbf{u})^{n+1}-(\rho \mathbf{u})^{n}}{\Delta t}=-\frac{1}{\varepsilon^{\alpha}}\left(\sum_{r} c_{j r} \widehat{\alpha}_{j r}\left(\widehat{I}_{d}-M_{r}\right)\right) \mathbf{u}_{j}^{n+1}
$$

Furthermore, we obtain

$$
-\left(\frac{\rho^{n+1}}{\Delta t}+\frac{1}{\varepsilon} \sum_{r} c_{j r} \widehat{\alpha}_{j r}\left(\widehat{I}_{d}-M_{r}\right)\right) \mathbf{u}_{j}^{n+1}=-\rho_{j}^{n} \mathbf{u}_{j}^{n}
$$

Assuming that the matrix $\sum_{r} c_{j r} \widehat{\alpha}_{j r}\left(\widehat{I}_{d}-M_{r}\right)$ is invertible and since $\rho_{j}^{n+1}=\rho_{j}^{n}$ and $\mathbf{u}_{j}^{n}=\mathbf{0}$ we conclude that $\mathbf{u}_{j}^{n+1}=\mathbf{0}$

This result shows (as in 1D) that the AP scheme preserves exactly the discrete hydrostatic steady states. Consequently for the continuous hydrostatic equilibrium, the numerical error is only governed by the truncation error between the discrete and continuous steady states. That is the reason why we propose to modify the scheme as previously to obtain an arbitrary high order discretization of the steady states.

\subsubsection{High-Order reconstruction of the equilibrium}

As in 1D the order of the spatial numerical error for a steady state is governed by the error between discrete and continuous steady state. The actual discrete steady state is defined by $\nabla_{r} p=-(\rho \nabla \phi)_{r}$ with $(\rho \nabla \phi)_{r}=\rho_{r} \nabla_{r} \phi$. Where $\rho_{r}$ is the average of the density around a node $r$ and $\nabla_{r} \phi$ the nodal gradient of $\phi$. To increase the accuracy and the order of the method we propose to modify the discrete steady state with a high order polynomial reconstruction of the steady state, as we did in 1D. The new steady state is given by $\nabla_{r} p=-(\rho \nabla \phi)_{r}^{H O}$ with

$$
(\rho \nabla \phi)_{r}^{H O}=\left(\frac{1}{V_{r}} \int_{V_{r}} \nabla p+\frac{1}{V_{r}} \int_{V_{r}} \rho \nabla \phi-\nabla_{r} p\right)
$$

with $\nabla_{r} p=-\left(\sum_{j} \widehat{\beta}_{j r}\right)^{-1} \sum_{j} p_{j} \mathbf{C}_{j r}$. To finish the method it is sufficient to find a good strategy to compute the polynomial interpolation of $p, \rho$ and $\phi$ and the integrals : $\int_{V_{r}} \nabla p$ and $\int_{V_{r}} \rho \nabla \phi$. We will focus now on a high-order reconstruction method, that we will use for $p, \rho$ or $\phi$, adapted to a 2D unstructured mesh. Therefore we have chosen to use the high order $k$-exact least-squares polynomial reconstruction methods. This method is not optimal and cannot preserve the monotony. To obtain these properties we can use an adaptation of the CENO methods proposed by L. Ivan and C. Groth [17] and has been adapted to 2D unstructured meshes by S. McDonald et al. [21, 22, 18]. When presenting the $k$-exact Reconstruction algorithm, it's necessary to cite T. J. Barth et al. [1, 2], and we refer the reader to those articles as well as [21] for more details. We denote $f$ the 
variable (typically $p, \rho$ or $\phi$ ), the reconstruction $f_{r}^{k}$ of the solution at the Volume control $r$ (area $\left.V_{r}\right)$ is defined by

$$
f_{r}^{k}(x, y)=\sum_{\substack{d_{1}=0 \\ d_{1}+d_{2} \leq k}}^{k} \sum_{d_{2}=0}^{k} D_{d_{1}, d_{2}}\left(x-x_{r}\right)^{d_{1}}\left(y-y_{r}\right)^{d_{2}}
$$

where $k$ is the order of the piecewise polynomial interpolant. The coefficients $D_{d_{1} d_{2}}$ are the unknowns in this definition. The number of unknowns, that we will denote $N$, depend on the order $k$ by the formula:

$$
N=\frac{(k+1)(k+2)}{2}
$$

For $k=1,2$ and 3 respectively we will have 3,6 and 10 unknowns.

In order to have an actual $k$-exact reconstruction, one has to satisfy three conditions: mean conservation, error of order $O\left(\Delta x^{k+1}\right)$ for the quantity, error of order $O\left(\Delta x^{k}\right)$ for the derivative of the quantity, i.e. exact reconstruction of polynomials of degree at most $k$ and finally, compact stencil support. On quadrangular meshes we use the two layers algorithm to find these neighbours. This algorithm is as follows: parting from a node $\mathbf{x}_{r}$, we choose the cells which contain the node $\mathbf{x}_{r}$. At this moment we have the first layer of cells and it is sufficient for $k=1$. To obtain the second layer we take each cell which contains a node of the cells associated with the first layer. For example in Cartesian mesh the first layer contained 4 cells and the two layers 16 cells. Now we want to construct the system. We define the stencil around the node $r$ named $S(r)$. The number of cells in the stencil is $M$. We assume that

$$
\frac{1}{\left|\Omega_{j}\right|} \int_{\Omega_{j}} f_{r}^{k}(x, y) \mathrm{d} A=f_{j} \Leftrightarrow \int_{\Omega_{j}} f_{r}^{k}(x, y) \mathrm{d} A=\left|\Omega_{j}\right| f_{j} \quad \forall j \in S(r)
$$

Now we use the definition of $f_{r}^{k}$ in this relation, to deduce that

$$
\sum_{\substack{d_{1}=0 \\ d_{1}+d_{2} \leq k}}^{k} \sum_{\substack{d_{2}=0 \\ d_{1}}}^{k} D_{d_{1}, d_{2}}\left(\int_{\Omega_{j}}\left(x-x_{r}\right)^{d_{1}}\left(y-y_{r}\right)^{d_{2}}\right) \mathrm{d} A=\left|\Omega_{j}\right| f_{j} \quad \forall j \in S(r)
$$

This equation generated an overdetermined system $\mathrm{A}_{\mathrm{r}} \mathbf{x}_{\mathrm{r}}-\mathbf{b}_{\mathrm{r}}=\mathrm{e}$ where the terms of matrix $A_{r}\left(\operatorname{dim}\left(A_{r}\right)=M \times N\right)$ can be precomputed as they will depend only on the mesh, besides, $\mathbf{b}_{r}$ is the average of the solution, and $e$ is the error of the least square method.

$$
A_{r}=\left(\begin{array}{ccccc}
\left(\widehat{x^{0} y^{1}}\right)_{1} & \ldots & \left(\widehat{x^{d 1} y^{d 2}}\right)_{1} & \ldots & \left(\widehat{x^{k} y^{0}}\right)_{1} \\
\vdots & & \vdots & & \vdots \\
\left(\widehat{x^{0} y^{1}}\right)_{j} & \ldots & \left(\widehat{x^{d 1} y^{d 2}}\right)_{j} & \ldots & \left(\widehat{x^{k} y^{0}}\right)_{j} \\
\vdots & & \vdots & & \vdots \\
\left(\widehat{x^{0} y^{1}}\right)_{M} & \ldots & \left(\widehat{x^{d 1} y^{d 2}}\right)_{M} & \ldots & \left.\widehat{\left(x^{k} y^{0}\right.}\right)_{M}
\end{array}\right) \quad \mathbf{b}_{r}=\left(\begin{array}{l}
\left|\Omega_{1}\right| f_{1} \\
\vdots \\
\left|\Omega_{j}\right| f_{j} \\
\vdots \\
\left|\Omega_{M}\right| f_{M}
\end{array}\right) \quad \mathbf{x}_{r}=\left(\begin{array}{l}
D_{0,1} \\
\vdots \\
D_{d_{1}, d_{2}} \\
\vdots \\
D_{k, 0}
\end{array}\right)
$$

with

$$
\left(\widehat{x^{d 1} y^{d 2}}\right)_{j}=\left(\int_{\Omega_{j}}\left(x-x_{r}\right)^{d_{1}}\left(y-y_{r}\right)^{d_{2}}\right)
$$

Now we want to focus on how to compute the elements of the matrix $A$, i.e. an approximation of 50 For this purpose, we will use a Gaussian quadrature for general quadrilateral elements, that we will describe on the next section. 
We use the same notations as before: $\Omega_{j}$ denotes the $j$-th cell, and $\left(x_{i}, y_{i}\right)$ with $i=1, \ldots, 4$ denotes the vertices in a counter-clockwise order. We assume, as it would be in most cases, that the cell -a polygon of unknown vertices- can be decomposed on quadrangular elements. Therefore, we will focus only on quadrature rules for quadrangular elements. Nonetheless, quadrature rules are usually defined on regular orthogonal squares (i.e. usually cells found in Cartesian meshes), so we decided to take the general approach from Finite Volumes schemes (see for example: 23]) which generalises the usual Gaussian quadrature rules to general quadrangular elements. The approach is based on transforming every element to a reference orthogonal cell, centered at the origin, which vertices are: $(-1,-1),(1,-1),(1,1)$ and $(-1,1)$. For the mapping (a.k.a transformation) we use the nodal shape functions for quadrilaterals:

$$
\left\{\begin{array}{l}
N_{1}(\varepsilon, \eta)=\frac{1}{4}(1-\varepsilon)(1-\eta) \\
N_{2}(\varepsilon, \eta)=\frac{1}{4}(1+\varepsilon)(1-\eta) \\
N_{3}(\varepsilon, \eta)=\frac{1}{4}(1+\varepsilon)(1+\eta) \\
N_{4}(\varepsilon, \eta)=\frac{1}{4}(1-\varepsilon)(1+\eta)
\end{array}\right.
$$

We can notice that if we pass the coordinates of the vertices of the reference element we will find $N_{i}\left(\varepsilon_{j}, \eta_{j}\right)=\delta_{i j}$. Now, it's easy to write the mapping that will transform the coordinates of the quadrature points on the standard element to our quadrangular cell:

$$
\left\{\begin{array}{l}
x=P(\varepsilon, \eta)=\sum_{i=1}^{4} x_{i} N_{i}(\varepsilon, \eta) \\
y=Q(\varepsilon, \eta)=\sum_{i=1}^{4} y_{i} N_{i}(\varepsilon, \eta)
\end{array}\right.
$$

Finally we introduce the jacobian matrix of such a transformation:

$$
J(\varepsilon, \eta)=\left(\begin{array}{ll}
\frac{\partial x}{\partial \varepsilon} & \frac{\partial y}{\partial \varepsilon} \\
\frac{\partial x}{\partial \eta} & \frac{\partial y}{\partial \eta}
\end{array}\right)
$$

The values of the jacobian function will only be needed on the quadrature points, so for a quadrature rule of order $N$, we only need to compute $N \times 4$ elements. Furthermore these values can be precomputed for every cell $\Omega_{j}$. Now we can write the general Gaussian quadrature formula of order $\mathrm{N}$, with gaussian points $\left(\varepsilon_{i}, \eta_{i}\right)$ and corresponding weights $w_{i}$ :

$$
\iint_{\Omega_{j}} F(x, y) \mathrm{d} x \mathrm{~d} y=\sum_{i=1}^{N} \sum_{j=1}^{N} w_{i} w_{j}\left|J\left(\varepsilon_{i}, \eta_{j}\right)\right| F\left(P\left(\varepsilon_{i}, \eta_{j}\right), Q\left(\varepsilon_{i}, \eta_{j}\right)\right)
$$

To solve equation 50 , we only need to plug $F(x, y)=\left(x-x_{r}\right)^{d_{1}}\left(y-y_{r}\right)^{d_{2}}$.

\subsection{Numerical results in $2 \mathrm{D}$}

In this section we propose some numerical test to validate the asymptotic preserving and wellbalanced properties for the scheme on unstructured meshes. To verify these properties we propose some meshes with different deformations 

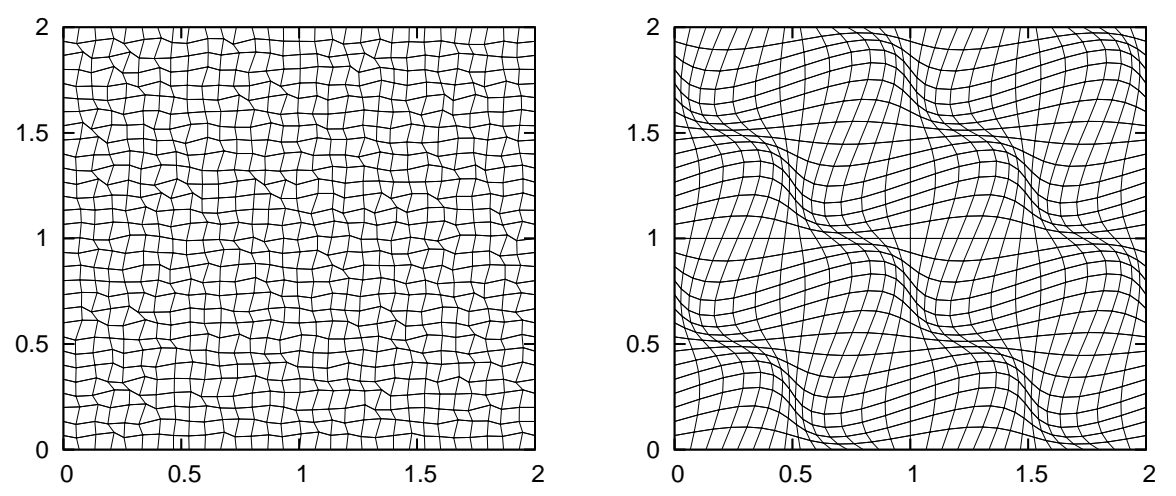

Figure 8: Unstructured quadrangular meshes (random and Collela meshes)

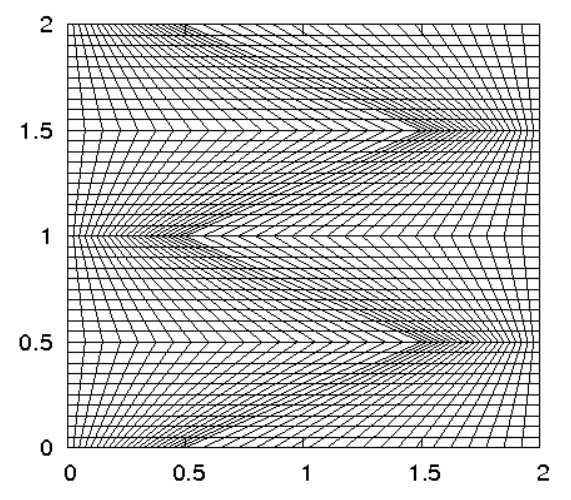

Figure 9: Kershaw mesh

\subsubsection{Numerical results for steady states solutions}

The LR AP scheme with $(\rho \nabla \phi)_{r}=\rho_{r}(\nabla \phi)_{r}$ where $\rho_{r}$ is an average of $\rho$ around the node $r$. The LR AP scheme with $q$ high order discretization of the steady states is called LR-AP O(q). We consider a domain $\Omega=[0,2]^{2}$ and the final time $T_{f}=0.1$. The difference test cases are given by

- Test case 1: Linear density: $\rho(t, \mathbf{x})=y+1, u(t, \mathbf{x})=0, p(t, \mathbf{x})=-\left(\frac{1}{2} y^{2}+y\right)+\left(\frac{1}{2} b^{2}+b\right) y+1$ and $\mathbf{g}=(0,-1)$.

- Test case 2: exponential density: $\rho(t, \mathbf{x})=e^{-(\mathbf{g}, \mathbf{x})}, u(t, \mathbf{x})=0, p(t, \mathbf{x})=e^{-(\mathbf{g}, \mathbf{x})}$ and $\mathrm{g}=(0,-1)$.

The classical scheme is called "LR", the AP scheme without higher order reconstruction is called "LR-AP", The AP scheme with the high order reconstruction of the equilibrium is called "LR-AP $\mathrm{O}(\mathrm{k})$ " with $k$ the order of polynomial reconstruction. With a polynomial reconstruction of degree $k$ for $p$ and $\rho$, we obtain a $k$-th order reconstruction since the order is $k+1$ for $\rho$ and $k$ for $\nabla p$.

The results are close to the 1D results. On Cartesian, Collela and Kershaw meshes, we observe that the classical scheme converges with the first order. Additionally, we can see that the AP scheme with $(\rho \nabla \phi)_{r}=\rho_{r} \nabla_{r} \phi$ converges with an order close to two and that we are exact for the discrete steady states (the discrete and continuous steady states are equal for constant density and linear density on Cartesian mesh). To finish we can also notice that when we use a second or third 




Table 5: Test case with linear density. The error corresponds to the $L_{1}$ error and $q$ to the order of convergence.

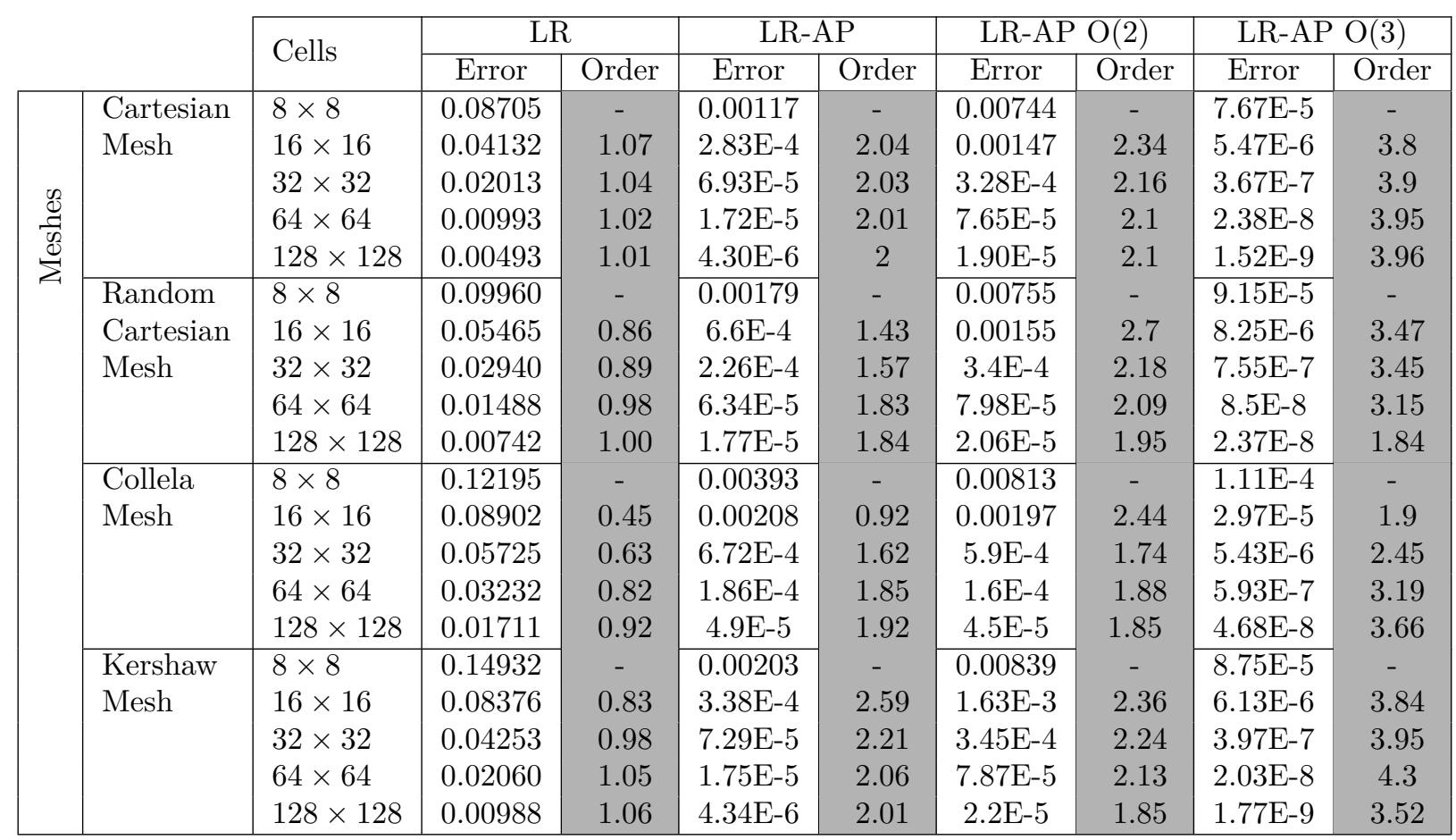

Table 6: Test case with exponential density. The error corresponds to the $L 1$ error and $q$ to the order of convergence. 
order reconstruction for $(\rho \nabla \phi)_{r}$ we converge with the third order at the end for these meshes. On random meshes, the situation is a little bit more complicated. We observe that the scheme with a second order reconstruction converges with second order, as expected. However, with the third order reconstruction, we remark that the order decreases on fine meshes. The reason of the problem is not clear. Our first idea to explain this, is that some cells become pathological and some small numerical instabilities appears. The second explanation is that the high order reconstruction is not completely robust on random grid. This problem does not exact is $1 \mathrm{D}$. In $1 \mathrm{D}$ for a $k$ order reconstruction we use $k+1$ cells to construction the linear problem which give the $k+1$ coefficients and this linear problem is solved exactly. In 2D we compute the coefficient of the polynomial reconstruction with a last square problem. Perhaps this is the approximation of the coefficients computed by the last square problem which generate the problem of convergence on fine grid on random meshes. Perhaps find a polynomial reconstruction which compute exactly the coefficients will be more efficient. The aim of this part is to prove that the idea to introduce a high order equilibrium in the scheme work. To our opinion, it is prove by these results however we can perhaps find a better way to construct the high order equilibrium on unstructured meshes.

Now we propose to study numerically the second subset of steady states (??) which correspond to high friction limit $(\alpha=0$ and $\beta=1)$. For this we present a Sod test case with $\varepsilon \geq 1$ and we will compare the classical LR scheme and the LR-AP scheme for different values of $\varepsilon$. The domain is $\Omega=[-1,1]^{2}$. The final time is $T_{f}=0.2$. The grid is a random grid. The initial data is given by

$$
\left\{\begin{array}{l}
\rho(t=0)=1, u(t=0)=0, p(t=0)=1, \text { for }\|\mathbf{x}\|<0.5 \\
\rho(t=0)=0.125, u(t=0)=0, p(t=0)=0.1, \text { for }\|\mathbf{x}\|>0.5
\end{array}\right.
$$

When $\sigma$ tends to the infinity, the stiff relaxation source term force is $\mathbf{u}=\mathbf{0}$ at the limit. Or, in other words, the initial solution is a stationary solution when $\sigma$ tends to the infinity.

\subsubsection{Numerical results for diffusion solutions}

To finish we propose to validate the scheme for the diffusion solutions (3) given by $\alpha=\beta=1$. For this we consider a Sod problem with $\sigma=1$ and $\varepsilon=0.005$. We compare the classical scheme on fine grid $(480 \times 480$ cells $)$ and coarse grid $(60 \times 60$ cells $)$ and the AP scheme on coarse grid.

We observe that the LR-AP scheme (Fig. 11) on coarse grid capture is less diffusive that the classical scheme (Fig. 12) on coarse grids if we compare to the fine solution (Fig. 13). Indeed we remark that the maximum of the solution is the same if we compare the solution of LR-AP scheme and the solution of the classical scheme on fine grid. We observe also that the classical scheme on coarse grid the solution have a larger support (because the scheme is more diffusive). After as in $1 \mathrm{D}$ the solution of the LR-AP scheme is probably better that the solution on fine grid. The same method is used to solve the $M_{1}$ model writhed as a Euler problem with stiff source term (radiative transfert problem) in [7] and [6]. For this case the diffusion limit is linear and we have analytical solutions. The results presented is these papers show that the AP scheme is convergent for the $M_{1}$ model. This is another argument to caddie the method but it be interesting to validate the method with analytical solutions.

\section{Conclusion}

In this paper, we have studied the design of an adapted finite volume scheme to treat the external forces source terms in the Euler equations on unstructured meshes. For this, we have coupled a Lagrange+remap nodal scheme (the fluxes are defined at the node on the mesh) with the JinLevermore method (AP technique). This method has been used previously with success for linear and simple non-linear models. At the end, we constructed a semi-implicit asymptotic preserving scheme for the friction source term and Well-Balanced scheme for the gravity term. Indeed we note that numerically the CFL condition is independent of $\varepsilon$ and that the scheme captures correctly 

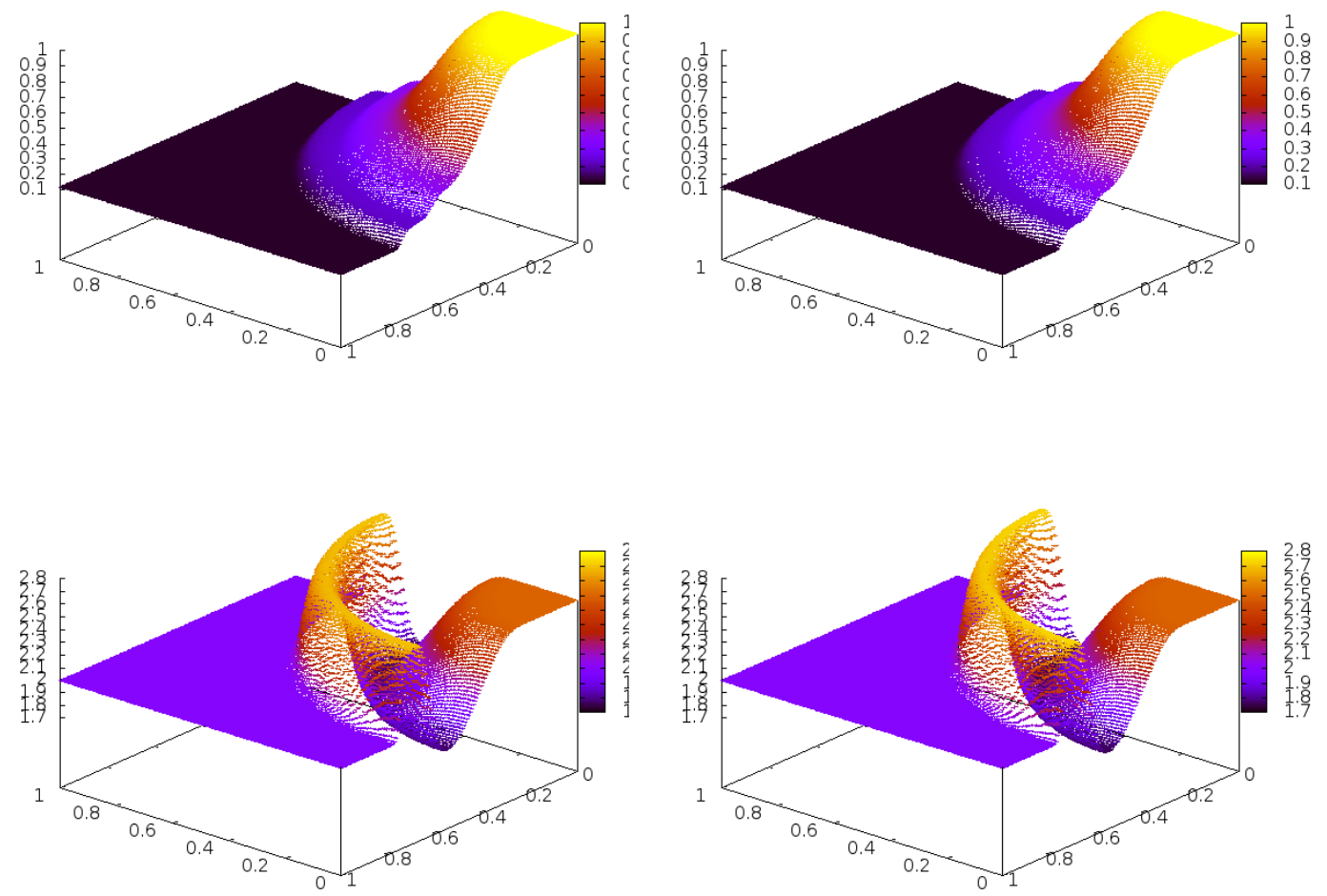

Figure 10: At the top: numerical density and internal energy given by the classical scheme. At the bottom: numerical density and internal energy given by the AP scheme. the results are given by $\varepsilon=1$ and 10000 cells

the asymptotic behavior associated with the friction term (unlike the the classical scheme). We don't have convergent results in the diffusion limit because we don't have an analytical solution of the limit diffusion scheme. However, some numerical results have shown that for a limit linear diffusion equation (Isothermal Euler equations with linear pressure or $M_{1}$ model [6]-[7]), the nonlinear diffusion limit scheme obtained converges on unstructured meshes with the same order that the advection scheme. In the future it be will interesting to verify if we obtain the same order for the non-linear limit diffusion equations.

Secondly, it is important to mention that the scheme respects the well-balanced property. The different numerical and theoretical results show that the scheme is well-balanced for the hydrostatic equilibrium because the discrete steady states at the interface are exactly captured. For a general steady state, our results have shown that, the order of convergence in space is only governed by the error between the discrete states and the continuous steady state. The formulation of our method allows us to modify easily the discrete steady states with a high order polynomial discretization to obtain at the end a first order scheme which preserves numerically the positivity and which converges with an arbitrary order around the steady states. In general the WB scheme for Euler equations and generic equilibrium converges with second order. Our method to increase the order is new in this context, simple and can be use for other well-balanced schemes. At the end we have obtained a scheme which allows us to capture correctly the behavior induced by the source terms, stiff and not stiff, without a large additional CPU cost on unstructured meshes. 

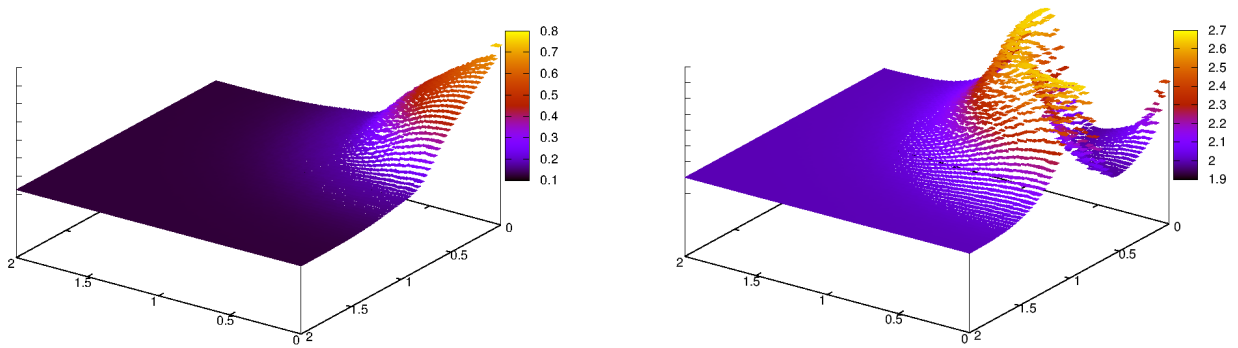

Figure 11: Density (left) and energy (right) for the classical LP scheme. Coarse grid.
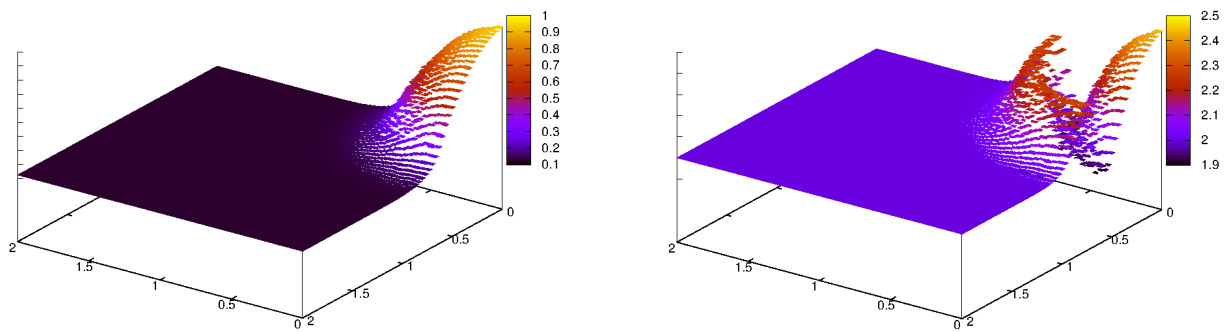

Figure 12: Density (left) and energy (right) for the LP AP scheme. Coarse grid.

\section{References}

[1] T. J. Barth and P. O. Fredrickson, Higher Order Solution of the Euler Equations on Unstructured Grids Using Quadratic Reconstruction, Paper 90-0013, AIAA, January 1990.

[2] T. J. Barth, Recent Developments in High Order K-Exact Reconstruction on Unstructured Meshes, Paper 93-0668, AIAA, January 1993

[3] C. Berthon, R. Turpault Asymptotic preserving HLL schemes Num. Method Partial Diff. Equations, accepted.

[4] C. Buet, B. Després, E. Franck Design of asymptotic preserving schemes for the hyperbolic heat equation on unstructured meshes, Numerish Mathematik, 2012, Volume 122, Issue 2, pp $227-278$.

[5] C. Buet, B. Després, E. Franck Asymptotic preserving schemes for Friedrichs systems with stiff relaxation on unstructured meshes: applications to the angular discretization models in linear transport, Journal Scientific Computing (online), april 204

[6] C. Buet, B. Després, E. Franck Asymptotic preserving scheme with maximum principle for non linear radiative transfer model on unstructured meshes, C.R. Acad. Sci., Paris, Sér. I, Math., Vol 350, N 11-12 P. 633-638, june 2012

[7] C. Buet, B. Després, E. Franck Asymptotic Preserving Finite Volumes Discretization For Non-Linear Moment Model On Unstructured Meshes, FVCA VI Problems and Perspectives, Springer Proceedings in Mathematics Volume 4, 2011, pp 467-474.

[8] C. Chalons, F. Coquel, E. Godlewski, P-A. Raviart, N. Seguin Godunov-type schemes for hyperbolic systems with parameter dependent source. The case of Euler system with friction, M3AS, volume 20(11), pages 2109-2166, 2010.

[9] C. Chalons, M. Girardin, S. Kokh Large time step asymptotic preserving numerical schemes for the gas dynamics equations with source terms, SIAM J. Sci. Comput., 35(6) 

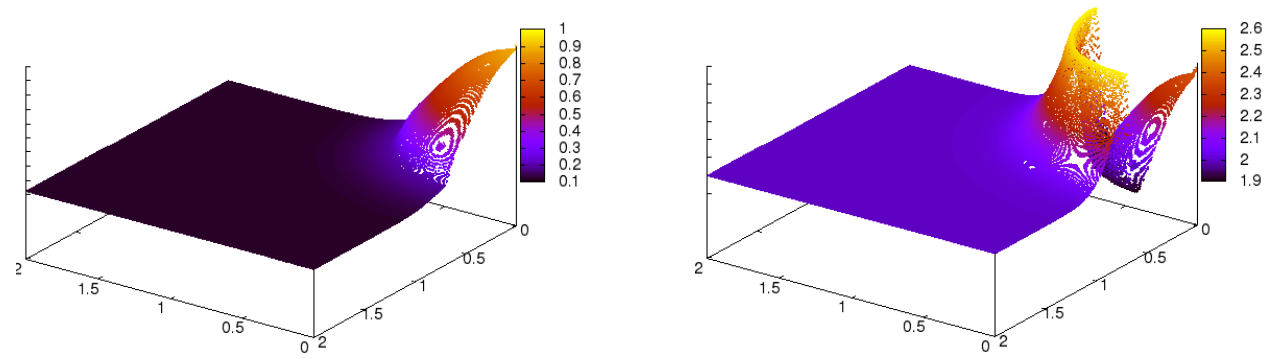

Figure 13: Density (left) and energy (right) for the classical LP scheme. Fine grid.

[10] G. Carré, S. Del Pino, B. Desprès, E. Labourasse A Cell-centered Lagrangian hydrodynamics scheme on general unstructured meshes in arbitrary dimension. JCP vol. 228 (2009) no14, pp. $5160-518$

[11] B. Després, C. Mazeran Symmetrization of Lagrangian gas dynamics and Lagrangian solvers, Arch. Ration. Mech. Anal. 178 (2005) 327-372. Sci

[12] E. Franck, Modified Finite Volume Nodal Scheme for Euler Equations with Gravity and Friction, FVCA VII-Methods and Theoretical Aspects Springer Proceedings in Mathematics and Statistics Volume 77, 2014, pp 285-292

[13] Greenberg J, Leroux A. Y. A well balanced scheme for the numerical processing of source terms in hyperbolic equations SIAM J. Numer. Anal - Vol 33 No 1, 1996.

[14] L. Gosse, G. Toscani An asymptotic-preserving well-balanced scheme for the hyperbolic heat equations C. R. Acad. Sci Paris,Ser. I 334 (2002) 337-342.

[15] L. Gosse, A well-balanced flux-vector splitting scheme designed for hyperbolic systems of conservation laws with source terms. Computers and Mathematics with Applications 39(9), $135 ? 159(2000)$

[16] P. C. Hammer, O. J. Marlowe, and A. H. Stroud; Numerical integration over simplexes and cones; Math Tabl. natn. Res. Coun., 10, 1956

[17] L. Ivan and C. P. T. Groth, High-Order Central ENO Finite-Volume Scheme with Adaptive Mesh Refinement, AIAA Paper 2007-4323, June 2007

[18] L. Ivan and C. P. T. Groth, High-Order Solution-Adaptive Central Essentially Non-Oscillatory (CENO) Method for Viscous Flows, Journal of Computational Physics, Vol. 257, pp. 830-862, 2013

[19] S. Jin, D. Levermore Numerical schemes for hyperbolic conservation laws with stiff relaxation terms. JCP 126,449-467,1996.

[20] J. Luo, K. Xu, N. Liu, A well-balanced symplecticity-preserving gas-kinetic scheme for hydrodynamic equations under gravitational field. SIAM Journal on Scientific Computing 33(5), $2356 ? 2381$ (2011)

[21] S. D. McDonald, Development of a high-order finite volume method for unstructured meshes, Master's thesis, University of Toronto, 2011

[22] S. D. McDonald, M. R. J. Charest, and C. P. T. Groth, High-Order CENO Finite-Volume Schemes for Multi-Block Unstructured Mesh, AIAA Paper 2011-3854, June 2011.

[23] B.A. Szabo and I. Babuška. Finite Element Analysis. Wiley-Interscience publication. Wiley, 1991 
[24] R. Natalini and M. Ribot, An asymptotic high order mass-preserving scheme for a hyperbolic model of chemotaxis, SIAM J. Numer. Anal. 50 (2012), no. 2, 883-905

[25] R. Natalini, M. Ribot, M. Twarogowska. A well-balanced numerical scheme for a one dimensional quasilinear hyperbolic model of chemotaxis, Commun. Math. Sci. 12 (2014), no.1, 13-39.

[26] M. Zenk, C. Berthon et C. Klingenberg, A well-balanced scheme for the Euler equations with a gravitational potential, FVCA VII-Methods and Theoretical Aspects Springer Proceedings in Mathematics and Statistics Volume 77, 2014 